\title{
MAPPINGS OF FINITE DISTORTION FROM GENERALIZED MANIFOLDS
}

\author{
VILLE KIRSILÄ
}

\begin{abstract}
We give a definition for mappings of finite distortion from a generalized manifold with controlled geometry to a Euclidean space. We prove that the basic properties of mappings of finite distortion are valid in this context. In particular, we show that under the same assumptions as in the Euclidean case, mappings of finite distortion are open and discrete.
\end{abstract}

\section{INTRODUCTION}

Mappings of finite distortion are generalizations of quasiregular mappings, defined as follows. Let $\Omega \subset \mathbb{R}^{n}$ be a domain. We say that $f \in W_{l o c}^{1,1}\left(\Omega, \mathbb{R}^{n}\right)$ is a mapping of finite distortion if the Jacobian determinant $J_{f} \in L_{l o c}^{1}(\Omega)$, and if there exists a measurable function $K: \Omega \rightarrow[1, \infty[$ such that

$$
|D f(x)|^{n} \leq K(x) J_{f}(x), \text { a.e. } x \in \Omega .
$$

Furthermore, $f$ is called quasiregular if the function $K$ is bounded. In the late $60 \mathrm{~s}$, Reshetnyak proved a fundamental result in the theory of quasiregular mappings, namely that a nonconstant quasiregular map is open and discrete. For mappings of finite distortion, a similar result was obtained by Iwaniec and Šverák in [15]. They showed for $n=2$ that Reshetnyak's result is still valid for a mapping of finite distortion $f \in W_{l o c}^{1,2}(\Omega)$, with $K \in L_{l o c}^{1}(\Omega)$. According to an example, given by Ball in [2], the result of Iwaniec and Sverák is sharp in the sense that for every $p<1$ there is a Lipschitz map with $K \in L_{l o c}^{p}(\Omega)$ that is not discrete. Later, in [18], Manfredi and Villamor proved that the result holds also in higher dimensions if $f \in W_{l o c}^{1, n}\left(\Omega, \mathbb{R}^{n}\right)$ and $K \in L_{l o c}^{p}(\Omega)$, for some $p>n-1$. Recently, Hencl and Rajala showed that the result of Manfredi and Villamor is sharp. In 14, they gave an example of a Lipschitz mapping in any dimension $n \geq 3$ with $K \in L^{n-1}(\Omega)$ that is not discrete. The main theorem of this paper is Theorem 3.3, which states that, under assumptions akin to the Euclidean case, a mapping of finite distortion from a generalized $n$-manifold with suitable controlled geometry to $n$-dimensional Euclidean space, is open and discrete. Our proof is new even in the Euclidean setting.

It is an interesting question when a given metric space is locally bi-Lipschitz equivalent to a Euclidean space. Motivated by this question, in [12, Heinonen and Rickman studied mappings of bounded length distortion. A sense-preserving, open and discrete map $f$ is called mapping of bounded length distortion, or BLD-map,

Received by the editors June 26, 2014 and, in revised form, October 13, 2014.

2010 Mathematics Subject Classification. Primary 30C65; Secondary 30L10.

The author was financially supported by the Finnish National Doctoral Programme in Mathematics and its Applications and by the Academy of Finland, project 257482. 
if it satisfies the bi-Lipschitz condition on rectifiable paths. That is,

$$
\frac{1}{C} \text { length } \gamma \leq \text { length } f \circ \gamma \leq C \text { length } \gamma
$$

for some constant $C>0$ and for every rectifiable path $\gamma$. BLD-maps can be seen as a generalization of bi-Lipschitz maps, but unlike a bi-Lipschitz map, a BLD-map does not need to be a homeomorphism. In [12, Heinonen and Rickman developed the basic theory of BLD-maps between generalized manifolds. Among other things, they introduced such geometric conditions on generalized manifolds that it was possible to give an analytic definition for BLD-maps. To be more precise, BLD-maps can be identified as those quasiregular maps that are locally Lipschitz and have Jacobian determinant bounded uniformly away from zero. In 13, Heinonen and Sullivan characterized metric spaces that are cohomology $n$-manifolds and admit local BLD-maps into $\mathbb{R}^{n}$. Moreover, in 8], Heinonen and Keith showed that an Ahlfors $n$-regular, locally linearly contractible homology $n$-manifold in $\mathbb{R}^{n}$ has local bi-Lipschitz parametrizations if it admits local Cartan-Whitney presentations in a certain Sobolev class. In fact, they gave an analytic characterization for topological manifolds among all the homology $n$-manifolds.

The purpose of this paper is to extend the theory of BLD-maps to quasiregular maps and to mappings of finite distortion. On the other hand, our results and methods can be useful also when studying if a given metric space is locally quasiconformally equivalent to a Euclidean space.

The definition of a generalized $n$-manifold allows us to use the theory of topological degree. In particular, it is possible to define sense-preserving maps. This is important, since many of our arguments are based on degree theory. As seen from the definition of mappings of finite distortion, the Jacobian determinant of such a map is non-negative almost everywhere. In the Euclidean case this assumption together with convenient analytic assumptions implies that the map is sense-preserving. We would like our notion of Jacobian to provide similar results.

We assume that our domain space $\mathcal{S}$ is an Ahlfors $n$-regular, $n$-rectifiable subset of $\mathbb{R}^{m}$, supporting a weak $(n-1)$-Poincaré inequality. In addition, we assume that $\mathcal{S}$ can be metrically oriented. This assumption can be replaced by a more geometric assumption that the space is linearly locally contractible. For a short discussion on that direction see Remark 2.35. In the definition of mappings of finite distortion, we use the notion of Newtonian spaces. Newtonian spaces are a generalization of Sobolev spaces to metric measure spaces. In our setting, mappings of finite distortion are approximately differentiable and the approximate derivative will serve as an upper gradient. For a comprehensive study of Newtonian spaces and upper gradients, see [11] and [25]. Our first step is to show that mappings of finite distortion are sense-preserving. Using this property, we are able to prove that they satisfy Lusin condition $(N)$, and are monotone and differentiable almost everywhere. For discreteness and openness, we prove that the preimage of a point has 1-dimensional Hausdorff measure zero. Here among other techniques the path lifting property of branched covers is used. When this is done, we are able to apply the theory of topological degree to conclude that our mappings are discrete and open. 


\section{Preliminaries}

Throughout this paper, the distance between elements $x$ and $y$ of a metric space is denoted by $|x-y|$.

\subsection{Newtonian spaces.}

Definition 2.1. Let $X$ and $Y$ be two metric spaces and $f: X \rightarrow Y$. We say that a Borel function $g: X \rightarrow[0, \infty]$ is an upper gradient of $f$ if

$$
|f(x)-f(y)| \leq \int_{\gamma} g d s
$$

for every locally rectifiable path $\gamma:[0,1] \rightarrow X$ with $\gamma(0)=x$ and $\gamma(1)=y$.

Let $\Gamma$ be a collection of rectifiable paths in a metric measure space $(X, \mu)$. For $1 \leq p<\infty$, the $p$-modulus of $\Gamma$ is defined by

$$
\operatorname{Mod}_{p}(\Gamma)=\inf \int_{X} g(x)^{p} d \mu(x) .
$$

Here the infimum is taken over all Borel functions $g$ satisfying $\int_{\gamma} g d s \geq 1$, for every $\gamma \in \Gamma$. Let $\Gamma_{A}$ be the collection of all rectifiable paths in $X$, and $p \geq 1$. We say that $g$ is a $p$-weak upper gradient of $f$, if there is $\Gamma_{0} \subset \Gamma_{A}$, such that $\operatorname{Mod}_{p}\left(\Gamma_{0}\right)=0$ and (11) holds for every $\gamma \in \Gamma_{A} \backslash \Gamma_{0}$. We use phrase "for $p$-almost every path", to say that some property holds for all paths excluding a set of $p$-modulus zero. It is not too difficult to see that if $\mu$ satisfies $\mu(B)<\infty$ for every ball $B \subset X$, and if $\operatorname{Mod}_{p} \Gamma=0$ for some $p \geq q \geq 1$, then also $\operatorname{Mod}_{q} \Gamma=0$. Thus under these assumptions, a $p$-weak upper gradient is also a $q$-weak upper gradient.

Definition 2.2. Let $(X, \mu)$ be a metric measure space. If a function $f \in L^{p}(X)$ has a $p$-integrable $p$-weak upper gradient, we say that $f \in N^{1, p}(X)$. Let $\left\{f_{i}\right\}_{i=1}^{\infty}$ be a sequence in $N^{1, p}(X)$. We say that it converges to $f \in N^{1, p}(X)$ in the Newtonian sense, or in $N^{1, p}(X)$, if there exist upper gradients $g_{i}$ of $f-f_{i}$, such that

$$
\int_{X}\left|f(x)-f_{i}(x)\right|^{p} d \mu(x)+\int_{X} g_{i}(x)^{p} d \mu(x) \rightarrow 0, \text { as } i \rightarrow \infty .
$$

Assume now that $f$ maps $X$ to $\mathbb{R}^{k}$. In case that every component function of $f$ belongs to the Newtonian space $N^{1, p}(X)$, we write $f \in N^{1, p}\left(X, \mathbb{R}^{k}\right)$. Convergence in $N^{1, p}\left(X, \mathbb{R}^{k}\right)$ is defined to be convergence of component functions in $N^{1, p}(X)$. Furthermore, we say that $f \in N_{l o c}^{1, p}(X)$ if $f$ has a $p$-weak upper gradient $g$, and $f, g \in L^{p}(\Omega)$, for every compact $\Omega \subset X$.

Definition 2.3. Let $Q>0$ be a real number. We say that a metric space $X$ endowed with a Borel measure $\mu$ is Ahlfors $Q$-regular if there exists a constant $C_{A} \geq 1$, such that

$$
\frac{1}{C_{A}} r^{Q} \leq \mu(B(x, r)) \leq C_{A} r^{Q},
$$

for every ball $B(x, r) \subset X$ satifying $r \leq \operatorname{diam} X$.

It is well known that if a locally compact metric space $X$ satisfies the Ahlfors $Q$-regularity condition with some Borel measure $\mu$ then $X$ equipped with the $Q$ dimensional Hausdorff measure is Ahlfors $Q$-regular as well. Furthermore, in this case $\mu$ is comparable with the Hausdorff measure. For this reason we always assume that each Ahlfors $Q$-regular space is equipped with the $Q$-dimensional Hausdorff measure. 
Definition 2.4. Let $(X, \mu)$ be a metric measure space and $p \geq 1$. We say that $(X, \mu)$ supports a weak $p$-Poincaré inequality if there exist constants $C_{P}$ and $\lambda>1$ such that for every $f \in L_{l o c}^{1}(X)$ we have

$$
f_{B}\left|f(x)-f_{B}\right| d \mu(x) \leq C_{p} \operatorname{diam}(B)\left(f_{\lambda B} g(x)^{p} d \mu(x)\right)^{\frac{1}{p}},
$$

whenever $g$ is a $p$-weak upper gradient of $f$, and $B \subset X$ is a ball satisfying $\operatorname{diam} B \leq$ $\operatorname{diam} X$. Here $f_{B}$ is the integral average $f_{B}=f_{B} f=\frac{1}{\mu(B)} \int_{B} f(x) d \mu(x)$. Note that by Hölder's inequality, a weak $p$-Poincaré inequality implies a weak $q$-Poincaré inequality for $q>p$. The converse does not hold in general.

Nowadays, it is a well-known fact that if $X$ is an Ahlfors n-regular metric measure space supporting a weak Poincaré inequality, then Lipschitz mappings forms a dense subset of $N^{1, n}(X)$. Actually, by a slight modification on the proof of [11, Theorem 7.2.1], we get

Theorem 2.5. Fix $n \geq 1$, and let $X$ be an Ahlfors $n$-regular metric space supporting a weak $n$-Poincaré inequality. For every function $f \in N^{1, n}(X)$, there is a sequence $\left\{f_{i}\right\}_{i=1}^{\infty}$ satisfying the following:

(1) $f_{i} \rightarrow f$, in $N^{1, n}(X)$, as $i \rightarrow \infty$.

(2) $\mathcal{H}^{n}\left(\left\{x: f_{i}(x) \neq f(x)\right\}\right) \leq \frac{1}{i}$, for every $i$.

(3) $\left\{x: f_{i+1}(x) \neq f(x)\right\} \subset\left\{x: f_{i}(x) \neq f(x)\right\}$, for every $i$.

(4) $f_{i}(x) \leq f_{i+1}(x) \leq f(x)$ for every $x \in X$ and for every $i$.

(5) Each $f_{i}$ is $C(i)$-Lipschitz continuous.

Next we introduce pointwise Lipschitz constants.

Definition 2.6. Let $X$ and $Y$ be two metric spaces. For continuous $f: X \rightarrow Y$ we define Borel functions

$$
\operatorname{Lip} f(x):=\limsup _{y \rightarrow x} \frac{|f(x)-f(y)|}{|x-y|}
$$

and

$$
\operatorname{lip} f(x):=\liminf _{r \rightarrow 0} \sup _{|x-y| \leq r} \frac{|f(x)-f(y)|}{r} .
$$

If $x$ is isolated we set $\operatorname{Lip} f(x)=\operatorname{lip} f(x)=0$. However, in our applications isolated points do not occur.

Remark 2.7. Trivially, $\operatorname{lip} f(x) \leq \operatorname{Lip} f(x)$ for every $x \in X$. It follows from 4, Theorem 4.38] that if $X$ is an Ahlfors $n$-regular metric measure space supporting a weak $p$-Poincaré inequality for some $1<p<\infty$, then for every locally Lipschitz map $f: X \rightarrow \mathbb{R}^{k}$ and for almost every $x \in X$

$$
\operatorname{Lip} f(x)=\operatorname{lip} f(x) .
$$

Furthermore, under these assumptions by [4, Proposition 4.26], there is a constant $C$ depending only on space $X$ such that for any $p$-integrable $p$-weak upper gradient $g$ of $f$ and for almost every $x \in \mathcal{S}$,

$$
\text { Lip } f(x) \leq C g(x) \text {. }
$$

On the other hand, $\operatorname{lip} f$ is an upper gradient for any locally Lipschitz map $f$ between two metric spaces. For the proof of this fact see [11, Lemma 5.2.6]. 
The following lemma shows that being an upper gradient is a local property. For the proof, see [11, Lemma 5.2.10].

Lemma 2.8. Let $(X, \mu)$ be a metric measure space and $Y$ a metric space. Suppose that $f: X \rightarrow Y$ is absolutely continuous on p-almost every path. Let $E$ be a Borel subset of $X$, and assume that there are maps $v, w: X \rightarrow Y$, such that $v=f$ almost everywhere on $E$ and $w=f$ almost everywhere on $X \backslash E$. If $v$ and $w$ possess p-integrable, $p$-weak upper gradients $\sigma$ and $\tau$, respectively, then the function

$$
\rho=\sigma \chi_{E}+\tau \chi_{E^{C}}
$$

is a p-integrable p-weak upper gradient of $f$.

Remark 2.9. If $X$ is a metric measure space, $Y$ a metric space and $f: X \rightarrow Y$ has a locally $p$-integrable $p$-weak upper gradient then $f$ is absolutely continuous on $p$-almost every path. For a proof of this fundamental fact; see [11, Proposition 5.3.3].

2.2. Rectifiable sets and approximate differentiability. In this section we study properties of rectifiable sets and give two concepts of differentiability. We also give a Rademacher-Stepanov type theorem. Our main references in this section are [5], [19] and [20].

Let $E \subset \mathbb{R}^{m}$ be a $\mathcal{H}^{n}$-measurable set and $x \in \mathbb{R}^{m}$. In the case that

$$
\lim _{r \rightarrow 0} \frac{\mathcal{H}^{n}(E \cap B(x, r))}{\omega_{n} r^{n}}
$$

exists, we call it the $n$-dimensional density of the set $E$ at point $x$. Here and in what follows, $\omega_{n}$ stands for the Lebesque measure of the unit ball in $\mathbb{R}^{n}$. It is well known that for a $\mathcal{H}^{n}$-measurable set $E \subset \mathbb{R}^{n}, \mathcal{H}^{n}$-almost every point $x \in E$ has density 1 . For sets with smaller dimension that does not hold in general. For instance, there is a Cantor type set $C \subset \mathbb{R}^{2}$ with positive $\mathcal{H}^{1}$-measure such that

$$
\limsup _{r \rightarrow 0} \frac{\mathcal{H}^{1}(C \cap B(x, r))}{\omega_{1} r}<1,
$$

for almost every $x \in C$. The Besicovitch-Marstrand-Mattila theorem [19, Theorem 17.6] states that so-called rectifiable sets are exactly the sets where this phenomenom cannot happen.

Definition 2.10. Let $\mathcal{S} \subset \mathbb{R}^{m}$ be a $\mathcal{H}^{n}$-measurable set, $n \leq m$, with locally finite $\mathcal{H}^{n}$-measure. We say that $\mathcal{S}$ is $n$-rectifiable if there are Lipschitz mappings $\phi_{i}: \mathbb{R}^{n} \rightarrow \mathbb{R}^{m}, i \in \mathbb{N}$, such that

$$
\mathcal{H}^{n}\left(\mathcal{S} \backslash \bigcup_{i=1}^{\infty} \phi_{i}\left(\mathbb{R}^{n}\right)\right)=0 .
$$

Theorem 2.11 (Besicovitch-Marstrand-Mattila). Let $E \subset \mathbb{R}^{m}$ be a Borel set with $\mathcal{H}^{n}(E)<\infty$. Then $E$ is n-rectifiable if and only if

for almost every $x \in E$.

$$
\lim _{r \rightarrow 0} \frac{\mathcal{H}^{n}(E \cap B(x, r))}{\omega_{n} r^{n}}=1,
$$

It is not too difficult to see that on an $n$-rectifiable set $\mathcal{H}^{n}$-almost every point has $n$-dimensional density 1 , but the converse is a deep result. The fact that almost every point of a rectifiable set can be associated with an approximate tangent plane 
is crucial for our work. Actually, existence of the tangent planes is another characterization of rectifiable sets. In this paper $n$-rectifiable sets are always assumed to be equipped with the $n$-dimensional Hausdorff measure. The following theorem is a direct consequence of [19, Definition 15.17] and [19, Theorem 15.19].

Theorem 2.12. Let $\mathcal{S}$ be an $n$-rectifiable subset of $\mathbb{R}^{m}$. For $\mathcal{H}^{n}$-almost every point $a \in \mathcal{S}$ there is a unique $n$-dimensional plane called approximate tangent plane $\operatorname{apTan}(a, \mathcal{S})$ satisfying

$$
\lim _{r \rightarrow 0} \frac{\mathcal{H}^{n}(\mathcal{S} \cap B(a, r) \backslash X(a, \operatorname{ap} \operatorname{Tan}(a, \mathcal{S}), s))}{\omega_{n} r^{n}}=0,
$$

for every $0<s<1$. Here

$$
X(a, V, s)=\left\{x \in \mathbb{R}^{m}: \operatorname{dist}(x, V)<s|x-a|\right\} .
$$

If one also assumes that the space is Ahlfors regular, the behaviour of the approximate tangent planes is even better. This is formulated in the following corollary.

Corollary 2.13. Let $\mathcal{S}$ be an n-rectifiable and Ahlfors $n$-regular subset of $\mathbb{R}^{m}$. Let $a \in \mathcal{S}$ such that apTan $(a, \mathcal{S})$ exists. For every $0<s<1$, there is $r>0$ such that

$$
\mathcal{S} \cap B(a, r) \backslash X(a, \operatorname{apTan}(a, \mathcal{S}), s)=\emptyset .
$$

Proof. Assume to the contrary that there is $s>0$ and a sequence $x_{i} \rightarrow a, x_{i} \in \mathcal{S}$ for every $i \in \mathbb{N}$, such that $\operatorname{dist}\left(x_{i}, \operatorname{ap} \operatorname{Tan}(a, \mathcal{S})\right) \geq s\left|x_{i}-a\right|$. In this case, we find balls $B\left(x_{i}, r_{i}\right)$ not intersecting $X\left(a, \operatorname{apTan}(a, \mathcal{S}), \frac{s}{2}\right)$. In fact, one can choose $r_{i}=$ $\left|x_{i}-a\right| \frac{s}{4}$. Hence,

$$
B\left(x_{i}, r_{i}\right) \subset B\left(a,\left|x_{i}-a\right|+r_{i}\right) \backslash X\left(\mathcal{S}, \operatorname{apTan}(a, \mathcal{S}), \frac{s}{2}\right),
$$

so Ahlfors regularity yields

$$
\begin{aligned}
& \lim _{r \rightarrow 0} \frac{\mathcal{H}^{n}\left(\mathcal{S} \cap B(a, r) \backslash X\left(a, \operatorname{apTan}(a, \mathcal{S}), \frac{s}{2}\right)\right)}{\omega_{n} r^{n}} \geq \lim _{i \rightarrow \infty} \frac{\mathcal{H}^{n}\left(\mathcal{S} \cap B\left(x_{i}, r_{i}\right)\right)}{\omega_{n}\left(\left|x_{i}-a\right|+r_{i}\right)^{n}} \\
& \geq \lim _{i \rightarrow \infty} \frac{\frac{1}{C_{A}} \frac{s^{n}}{4^{n}}\left|x_{i}-a\right|^{n}}{\omega_{n}\left(1+\frac{s}{4}\right)^{n}\left|x_{i}-a\right|^{n}}=\frac{\frac{1}{C_{A}} \frac{s^{n}}{4^{n}}}{\omega_{n}\left(1+\frac{s}{4}\right)^{n}}>0 .
\end{aligned}
$$

This contradicts Theorem 2.12 ,

In what follows, we will use the following notation. In the case that $\mathcal{S} \subset \mathbb{R}^{m}$ is an $n$-rectifiable set and $x \in \mathcal{S}$ such that $\operatorname{apTan}(x, \mathcal{S})$ exists, we write

$$
\pi_{x}: \mathcal{S} \rightarrow \operatorname{apTan}(x, \mathcal{S})
$$

for the restriction of the orthogonal projection $\pi: \mathbb{R}^{m} \rightarrow \operatorname{ap} \operatorname{Tan}(x, \mathcal{S})$. If $x \in \mathbb{R}^{m}$ and $r>0$ we denote

$$
\mathcal{S}^{n-1}(x, r)=\left\{y \in \mathbb{R}^{m}:|x-y|=r\right\} .
$$

Remark 2.14. Let $\mathcal{S}$ be an $n$-rectifiable, Ahlfors $n$-regular subset of $\mathbb{R}^{m}$, and let $x \in \mathcal{S}$ such that $\operatorname{ap} \operatorname{Tan}(x, \mathcal{S})$ exists. There is $r>0$, such that

$$
\mathcal{S} \cap \pi_{x}^{-1}(x) \cap B(x, r)=\{x\} .
$$

This can be seen as follows. According to Corollary 2.13, for $r>0$ small enough,

$$
\mathcal{S} \cap B(x, r) \subset X\left(x, \operatorname{apTan}(x, \mathcal{S}), \frac{1}{2}\right) .
$$


On the other hand,

$$
\pi_{x}^{-1}(x) \cap X\left(x, \operatorname{ap} \operatorname{Tan}(x, \mathcal{S}), \frac{1}{2}\right)=\{x\} .
$$

Thus $\pi_{x}^{-1}(x) \cap B(x, r) \subset\{x\}$. The opposite inclusion is clear.

Next we introduce two concepts related to differentiability. The first one, approximate differentiability, does not see sets of density zero.

Definition 2.15. Let $\mathcal{S} \subset \mathbb{R}^{m}$ be an $n$-rectifiable set and $f: \mathcal{S} \rightarrow \mathbb{R}^{k}$. We say that $f$ is approximately differentiable at $a \in \mathcal{S}$, if $\operatorname{ap} \operatorname{Tan}(a, \mathcal{S})$ exists and if there is a map $g: \mathbb{R}^{m} \rightarrow \mathbb{R}^{k}$, which is differentiable at $a$, satisfying

$$
\lim _{r \rightarrow 0} \frac{\mathcal{H}^{n}(\{x \in \mathcal{S}: f(x) \neq g(x)\} \cap B(a, r))}{\omega_{n} r^{n}}=0 .
$$

The restriction of $D g(a)$ to the shifted approximate tangent plane $\operatorname{apTan}(a, \mathcal{S})-a$ is called approximate differential of $f$ at $a$ and denoted by $a p D f(a)$. That is,

$$
\operatorname{apD} f(a): \operatorname{apTan}(a, \mathcal{S})-a \rightarrow \mathbb{R}^{k}, \operatorname{apD} f(a)=D g(a)_{\mid \operatorname{apTan}(a, \mathcal{S})-a} .
$$

Note that if $f$ and $g$ are approximately differentiable at $a$ and $\lambda \in \mathbb{R}$, then

$$
a p D(\lambda(f+g))(a)=\lambda a p D f(a)+\lambda a p D g(a) .
$$

The following theorem is part of [5, Theorem 3.2.19].

Theorem 2.16. Let $\mathcal{S} \subset \mathbb{R}^{m}$ be $n$-rectifiable, and $f: \mathcal{S} \rightarrow \mathbb{R}^{k}$ a Lipschitz map. Then $f$ is approximately differentiable $\mathcal{H}^{n}$-almost everywhere.

Corollary 2.17. Let $\mathcal{S} \subset \mathbb{R}^{m}$ be an $n$-rectifiable Ahlfors $n$-regular set supporting a weak $n$-Poincaré inequality. Then each $f \in N^{1, n}\left(\mathcal{S}, \mathbb{R}^{k}\right)$ is approximately differentiable $\mathcal{H}^{n}$-almost everywhere.

Proof. It follows from Theorem 2.5 that for each $i \in \mathbb{N}$ there is a Lipschitz map $f_{i}: \mathcal{S} \rightarrow \mathbb{R}^{k}$ such that for the set

$$
E_{i}=\left\{x \in \mathcal{S}: f_{i}(x) \neq f(x)\right\}
$$

we have $\mathcal{H}^{n}\left(E_{i}\right) \leq \frac{1}{i}$. Furthermore, $E_{i+1} \subset E_{i}$ for each $i \in \mathbb{N}$. Let

$$
P=\left\{x \in \mathcal{S}: a p D f_{i}(x) \text { does not exist for some } i \in \mathbb{N}\right\} .
$$

By Theorem 2.16 $\mathcal{H}^{n}(P)=0$ and thus $\mathcal{H}^{n}\left(\bigcap_{i} E_{i} \cup P\right)=0$. Let $x \in \mathcal{S} \backslash\left(\bigcap_{i} E_{i} \cup P\right)$. Then there is $i \in \mathbb{N}$ such that $x \in E_{i}^{c}$ and $f_{i}$ is approximately differentiable at $x$. Hence there is $g: \mathbb{R}^{m} \rightarrow \mathbb{R}^{k}$ such that $g$ is differentiable at $x$ and

$$
\lim _{r \rightarrow 0} \frac{\mathcal{H}^{n}\left(\left\{y \in \mathcal{S}: f_{i}(y) \neq g(y)\right\} \cap B(x, r)\right)}{\omega_{n} r^{n}}=0 .
$$

Because for $\mathcal{H}^{n}$-almost every $x \in E_{i}^{c} \cap \mathcal{S}$,

$$
\lim _{r \rightarrow 0} \frac{\mathcal{H}^{n}\left(E_{i} \cap B(x, r)\right)}{\omega_{n} r^{n}}=0
$$

(see [19, Theorem 6.2]), we may assume that (3) holds for $x$. Hence

$$
\begin{aligned}
& \lim _{r \rightarrow 0} \frac{\mathcal{H}^{n}(\{y \in \mathcal{S}: f(y) \neq g(y)\} \cap B(x, r))}{\omega_{n} r^{n}} \\
& \leq \lim _{r \rightarrow 0} \frac{\mathcal{H}^{n}\left(\left\{y \in \mathcal{S}: f_{i}(y) \neq g(y)\right\} \cap B(x, r) \cap E_{i}^{c}\right)}{\omega_{n} r^{n}}+\lim _{r \rightarrow 0} \frac{\mathcal{H}^{n}\left(E_{i} \cap B(x, r)\right)}{\omega_{n} r^{n}} \\
& =0 .
\end{aligned}
$$


By the definition of approximate differentiability, this proves the claim. For later use, notice that for $\mathcal{H}^{n}$-almost every $x \in \mathcal{S} \backslash \bigcap_{i} E_{i}$ there is $i \in \mathbb{N}$ such that $\operatorname{apD} f(x)=a p D f_{i}(x)$.

The assumption of Ahlfors regularity leads to a stronger differentiability result.

Definition 2.18. Let $\mathcal{S} \subset \mathbb{R}^{m}$ be an $n$-rectifiable set. We say that $f: \mathcal{S} \rightarrow \mathbb{R}^{k}$ is differentiable at $a \in \mathcal{S}$, if $\operatorname{ap} \operatorname{Tan}(a, \mathcal{S})$ exists and if there is a map $g: \mathbb{R}^{m} \rightarrow \mathbb{R}^{k}$, which is differentiable at $a$, and coincides with $f$ on $\mathcal{S}$. The restriction of $D g(a)$ to the shifted approximate tangent plane $\operatorname{ap} \operatorname{Tan}(a, \mathcal{S})-a$ is called differential of $f$ at $a$ and denoted by $D f(a)$. That is,

$$
D f(a): \operatorname{apTan}(a, \mathcal{S})-a \rightarrow \mathbb{R}^{k}, D f(a)=D g(a)_{\mid a p T a n(a, \mathcal{S})-a} .
$$

It is clear that if $f$ is differentiable at $a \in \mathcal{S}$, then $f$ is also approximately differentiable and $D f(a)=a p D f(a)$. Furthermore, differentiability implies continuity.

Theorem 2.19 (Rademacher-Stepanov Theorem). Let $\mathcal{S} \subset \mathbb{R}^{m}$ be an $n$-rectifiable and Ahlfors $n$-regular set. A mapping $f: \mathcal{S} \rightarrow \mathbb{R}^{k}$ is differentiable $\mathcal{H}^{n}$-almost everywhere in the set

$$
\left\{x \in \mathcal{S}: \lim _{r \rightarrow 0} \sup _{|x-y| \leq r} \frac{|f(x)-f(y)|}{|x-y|}<\infty\right\} .
$$

Theorem 2.19 can be proved in the same way as the Rademacher-Stepanov theorem is proved in [17]. In order to generalize the proof given in [17] two key facts are needed. First, Lipschitz mappings are required to be differentiable almost everywhere. Second, if $u$ and $v$ are two Lipschitz mappings satisfying $u \leq v$, then $D u(x)$ and $D v(x)$ should coincide for almost every point $x$ satisfying $u(x)=v(x)$. For the reader's convenience we prove the differentiability result for Lipschitz mappings and record from [3] that for a Lipschitz mapping $f$ we have $D f(x)=0 \mathcal{H}^{n}$-almost everywhere in the set $f^{-1}(0)$. These two facts guarantee that the proof given in 17 . passes through also in our setting. For a similar proof of a Rademacher-Stepanov Theorem see [3].

Lemma 2.20. Let $\mathcal{S} \subset \mathbb{R}^{m}$ be an $n$-rectifiable and Ahlfors $n$-regular set. Let $f: \mathcal{S} \rightarrow \mathbb{R}^{k}$ be locally Lipschitz. Then $f$ is differentiable almost everywhere.

Proof. We may assume that $f$ is Lipschitz. Assume that $f$ is approximately differentiable at $a \in \mathcal{S}$. By Definition 2.15 there is map $g: \mathbb{R}^{m} \rightarrow \mathbb{R}^{k}$ that is differentiable at $a$ and satisfies

$$
\lim _{r \rightarrow 0} \frac{\mathcal{H}^{n}(\{x \in \mathcal{S}: f(x) \neq g(x)\} \cap B(a, r))}{\omega_{n} r^{n}}=0 .
$$

First we will show that for every $\epsilon>0$ there exist $r_{0}>0$ such that

$$
|f(a)-f(x)-D g(a)(x-a)|<\epsilon|x-a|,
$$

whenever $x \in \mathcal{S} \cap B\left(a, r_{0}\right)$. Fix $\epsilon>0$, denote the Lipschitz constant associated to $f$ by $L$ and let $\rho=\frac{\epsilon}{1+L+\|D g(a)\|}$. Here $\|D g(a)\|$ is the operator norm of the differential matrix $D g(a)$. We may assume that $\rho<1$. Because both $f$ and $g$ are continuous at $a$ and because (44) holds, it is not too difficult to see that

$$
f(a)=\lim _{r \rightarrow 0} f_{B(a, r) \cap \mathcal{S}} f(x) d \mathcal{H}^{n}(x)=\lim _{r \rightarrow 0} f_{B(a, r) \cap \mathcal{S}} g(x) d \mathcal{H}^{n}(x)=g(a) .
$$


Since $g$ is differentiable at $a$, this together with (44) shows that there is $\delta>0$ such that

for any $0<r<\delta$. Here

$$
\mathcal{H}^{n}\left(P_{\epsilon} \cap B(a, r)\right)<\frac{1}{2 C_{A}} \rho^{n} r^{n},
$$

$$
P_{\epsilon}=\{x \in \mathcal{S}:|f(a)-f(x)-D g(a)(x-a)|>\epsilon|x-a|\} .
$$

Let $r_{0}=\frac{\delta}{2}$ and fix $x \in B\left(a, r_{0}\right)$. Because $\rho<1$ we have $B(x, \rho|x-a|) \subset$ $B(a, 2|x-a|)$. Here $2|x-a| \leq \delta$ and thus

$$
\mathcal{H}^{n}\left(P_{\epsilon} \cap B(a, 2|x-a|)\right) \leq \frac{1}{2 C_{A}} \rho^{n}|x-a|^{n} .
$$

On the other hand, by Ahlfors regularity

$$
\mathcal{H}^{n}(\mathcal{S} \cap B(x, \rho|x-a|)) \geq \frac{1}{C_{A}} \rho^{n}|x-a|^{n} .
$$

Thus there exist $z \in P_{\epsilon}^{c} \cap B(x, \rho|x-a|) \cap \mathcal{S}$. We have

$$
\begin{aligned}
& |f(a)-f(x)-D g(a)(x-a)| \\
& \leq|f(a)-f(z)-D g(a)(z-a)+f(z)-f(x)+D g(a)(z-a)-D g(a)(x-a)| \\
& \leq \epsilon|z-a|+(L+\|D g(a)\|)|x-z| \\
& \leq \epsilon|x-a|+(\epsilon+L+\|D g(a)\|)|x-z| \\
& \leq \epsilon|x-a|+(\epsilon+L+\|D g(a)\|) \rho|x-a| \leq 2 \epsilon|x-a| .
\end{aligned}
$$

We conclude that (5) holds. Hence the map $h: \mathbb{R}^{m} \rightarrow \mathbb{R}^{k}$,

$$
h(x)= \begin{cases}f(x) & \text { if } x \in \mathcal{S}, \\ D g(a)(x-a)+f(a) & \text { if } x \in \mathbb{R}^{m} \backslash \mathcal{S},\end{cases}
$$

is differentiable at $a$ and coincides with $f$ at $\mathcal{S}$. Since $f$ is approximately differentiable almost everywhere this proves the lemma.

The following lemma is a trivial consequence of [3, Proposition 2.9].

Lemma 2.21. Let $\mathcal{S}$ be an $n$-rectifiable and Ahlfors $n$-regular set and $f: \mathcal{S} \rightarrow \mathbb{R}^{k}$ a Lipschitz map. Then

$$
\lim _{r \rightarrow 0} \sup _{y \in B(x, r)} \frac{|f(x)-f(y)|}{|x-y|}=0
$$

and thus also $D f(x)=0$, for almost every $x \in f^{-1}(0)$.

Theorem 2.19 can now be proven using Lemma 2.20 and Lemma 2.21 as in 17; see also [3].

Definition 2.22. Let $\mathcal{S}$ be an $n$-rectifiable set in $\mathbb{R}^{m}$, and let $f: \mathcal{S} \rightarrow \mathbb{R}^{k}$. If $f$ is approximately differentiable at $x \in \mathcal{S}$ we define the generalized approximate Jacobian of $f$ denoted by $J_{f}(x)$, as follows. Assume first that an orthonormal basis of the $n$-dimensional vector space $\operatorname{ap} \operatorname{Tan}(x, \mathcal{S})-x$ is fixed.

- If $n<k$ we set $J_{f}(x)$ to be the square root of the sum of the squares of determinants of $n \times n$ submatrices of $\operatorname{apD} f(x)$.

- If $n=k$ we set $J_{f}(x)$ to be the determinant of $a p D f(x)$.

- If $n>k$ we set $J_{f}(x)$ to be the square root the of sum of the squares of determinant of $k \times k$ submatrices of $a p D f(x)$. 
The choice of the orthonormal basis of the shifted tangent planes is explained in Section 2.4. Note that in the case $n \neq k$ the Jacobian is always non-negative but in the case $n=k$ the Jacobian can be negative as well. The following coarea formula is stated in greater generality in [5, Theorem 3.2.22].

Theorem 2.23. Let $k \leq n$, and let $\mathcal{S}$ be an $n$-rectifiable set in $\mathbb{R}^{m}$. Consider a Lipschitz function $f: \mathcal{S} \rightarrow \mathbb{R}^{k}$. Then for every integrable function $g: \mathcal{S} \rightarrow \mathbb{R}$,

$$
\int_{\mathcal{S}} g(x)\left|J_{f}(x)\right| d \mathcal{H}^{n}(x)=\int_{\mathbb{R}^{m}} \int_{f^{-1}(y)} g(x) d \mathcal{H}^{n-k}(x) d \mathcal{H}^{k}(y) .
$$

2.3. Approximate derivatives and upper gradients. In this section we consider the connection between upper gradients and approximate derivatives. We will see that convergence in the Newtonian sense implies convergence of approximate differentials and thus convergence of the Jacobian determinants. This is important because we want that convergence in the Newtonian sense also carries topological information contained in the Jacobian determinant.

Lemma 2.24. Let $\mathcal{S}$ be an $n$-rectifiable Alhfors $n$-regular subset of $\mathbb{R}^{m}$. Assume that $f: \mathcal{S} \rightarrow \mathbb{R}^{k}$ is approximately differentiable $\mathcal{H}^{n}$-almost everywhere. Then

$$
\sup _{|y|=1}|\operatorname{apD} f(x) y|=\limsup _{y \rightarrow x}\left|\operatorname{apD} f(x) \frac{\left(\pi_{x} y-x\right)}{|x-y|}\right|
$$

for $\mathcal{H}^{n}$-almost every $x \in \mathcal{S}$.

Proof. Assume that $f$ is approximately differentiable at $x \in \mathcal{S}$. Since $\left|x-\pi_{x}(y)\right| \leq$ $|x-y|$ it is clear that

$$
\sup _{|y|=1}|\operatorname{apD} f(x) y| \geq \limsup _{y \rightarrow x}\left|\operatorname{apD} f(x) \frac{\left(\pi_{x} y-x\right)}{|x-y|}\right|
$$

for almost every $x \in \mathcal{S}$. Now assume to the contrary that equality does not hold for some $x \in \mathcal{S}$ which is a point of approximate differentiability of $f$. For simplicity we may assume that $x=0$. Then there is $z \in \operatorname{apTan}(0, \mathcal{S}),|z|=1$ with

$$
|\operatorname{apD} f(0) z|>\limsup _{y \rightarrow 0}\left|\operatorname{apD} f(0) \frac{\left(\pi_{0} y\right)}{|y|}\right| .
$$

It follows that there is actually a cone $C$ in $\operatorname{ap} \operatorname{Tan}(0, \mathcal{S})$ with vertex at the origin and such that

$$
\left|\operatorname{apD} f(0) \frac{z}{|z|}\right|>\limsup _{y \rightarrow 0}\left|\operatorname{apD} f(0) \frac{\left(\pi_{0} y\right)}{|y|}\right|
$$

for every $z \in C$. By Corollary $2.13 \limsup _{y \rightarrow 0} \frac{|y|}{\left|\pi_{0} y\right|}=1$ and thus

$$
\limsup _{y \rightarrow 0}\left|\operatorname{apD} f(0) \frac{\left(\pi_{0} y\right)}{|y|}\right|=\underset{y \rightarrow 0}{\limsup }\left|\operatorname{apDf(0)} \frac{\left(\pi_{0} y\right)}{\left|\pi_{0} y\right|}\right| \text {. }
$$

Therefore, by the counter-assumption there is $r_{0}>0$ such that

$$
C \cap \pi_{0} B\left(0, r_{0}\right)=\emptyset \text {. }
$$

This leads to a contradiction as follows. There is a line segment $L$ in the cone $C$, connecting the origin to the set $\{x \in \operatorname{ap} \operatorname{Tan}(0, \mathcal{S}):|x|=1\}$, and a positive number $\eta<\frac{1}{100}$ such that

$$
B\left(\frac{1}{2} s, 2 \eta|s|\right) \cap \operatorname{apTan}(0, \mathcal{S}) \subset C,
$$


whenever $s \in L$. According to [19, Theorem 15.11], there exist $0<r_{1}<\frac{1}{2} r_{0}$ and $\lambda>0$ such that

$$
\mathcal{H}^{n}\left(\mathcal{S} \cap B\left(\frac{1}{2} s, \eta|s|\right)\right) \geq \lambda|s|^{n},
$$

whenever $s \in L \cap B\left(0, r_{1}\right)$. In particular, for every $s \in L \cap B\left(0, r_{1}\right)$, there is $z_{s} \in$ $\mathcal{S} \cap B\left(\frac{1}{2} s, \eta|s|\right)$. Clearly $z_{s} \in B\left(0, r_{0}\right) \cap \mathcal{S}$ for every $s \in L \cap B\left(0, r_{1}\right)$. In order to show that if $s \in L \cap B\left(0, r_{1}\right)$ then $\pi_{0} z_{s} \in C \cap \pi_{0} B\left(0, r_{0}\right)$ we need to verify that $\pi_{0} z_{s} \in C$. We may assume that $r_{0}$ is chosen to be so small that

$$
\left|\pi_{0} z-z\right| \leq \eta\left|\pi_{0} z\right| \leq \eta|z|,
$$

whenever $z \in B\left(0, r_{0}\right)$. Under this assumption,

$$
\begin{aligned}
\left|\pi_{0} z_{s}-\frac{1}{2} s\right| & \leq\left|\pi_{0} z_{s}-z_{s}\right|+\left|z_{s}-\frac{1}{2} s\right| \leq \eta\left|z_{s}\right|+\eta|s| \leq \eta\left(\frac{1}{2}|s|+\eta|s|\right)+\eta|s| \\
& \leq 2 \eta|s| .
\end{aligned}
$$

Thus $\pi_{0} z_{s} \in B\left(\frac{1}{2} s, 2 \eta|s|\right) \cap \operatorname{apTan}(0, \mathcal{S}) \subset C$. This gives

$$
\pi_{0} z_{s} \in C \cap \pi_{0}\left(B\left(0, r_{0}\right)\right),
$$

which contradicts (6).

Remark 2.25. Since differentiability implies approximate differentiability, the lemma above applies also if $f$ is differentiable almost everywhere.

Definition 2.26. Let $\mathcal{S} \subset \mathbb{R}^{m}$ be an $n$-rectifiable set and let $f: \mathcal{S} \rightarrow \mathbb{R}^{k}$. Let $P_{f} \subset$ $\mathcal{S}$ be the smallest Borel set containing all the points where $f$ is not differentiable or for which

$$
\sup _{|y|=1}|D f(x) y| \neq \limsup _{y \rightarrow x}\left|D f(x) \frac{\left(\pi_{x} y-x\right)}{|x-y|}\right| .
$$

We define the norm of the differential to be $\|D f(\cdot)\|: \mathcal{S} \rightarrow[0, \infty]$,

$$
\|D f(x)\|= \begin{cases}\sup _{|y|=1}|D f(x) y| & \text { if } x \in \mathcal{S} \backslash P_{f}, \\ \infty & \text { if } x \in P_{f} .\end{cases}
$$

If $f$ is approximately differentiable $\mathcal{H}^{n}$-almost eveywhere, we define the function $\|$ apD $f(\cdot) \|$ similarily.

Remark 2.27. The norm of the differential is defined even if $f$ is nowhere differentiable. However, this convention serves our purposes. Since the $n$-dimensional Hausdorff measure is Borel regular it follows from Lemma 2.24 that if $\mathcal{S}$ is assumed to be Ahlfors $n$-regular and if $f$ is differentiable almost everywhere then $\mathcal{H}^{n}\left(P_{f}\right)=0$.

From now on, $\mathcal{S}$ will denote an $n$-rectifiable, Ahlfors $n$-regular metric measure space supporting a weak $n$-Poincaré inequality. As above, measure refers to the $n$-dimensional Hausdorff measure.

Lemma 2.28. Let $f: \mathcal{S} \rightarrow \mathbb{R}^{k}$. The function $\|D f(x)\|$ enjoys the following properties.

(1) $\|D f(x)\|=\operatorname{Lip} f(x)$ for every $x \in \mathcal{S} \backslash P_{f}$.

(2) If $f$ is continuous $\|D f(\cdot)\|$ is a Borel function. 
(3) If $f$ is locally Lipschitz then $\|D f(\cdot)\|$ is an upper gradient of $f$ and there is constant $C$ such that for any locally integrable $n$-weak upper gradient $h$ of $f$ and for almost every $x \in \mathcal{S}$,

$$
\|D f(x)\| \leq C h(x) .
$$

(4) If $f$ is continuous and $f \in N^{1, p}(\mathcal{S})$ for some $p \geq 1$, then $\|D f(\cdot)\|$ is a $p$-weak upper gradient of $f$.

Proof. Assume first only that $f: \mathcal{S} \rightarrow \mathbb{R}^{k}$. Fix $x \in \mathcal{S} \backslash P_{f}$. Then $f$ is differentiable at $x$. Let $g$ be as in the Definition 2.18, Then

$$
\begin{gathered}
\operatorname{Lip} f(x)=\limsup _{y \rightarrow x} \frac{|f(x)-f(y)|}{|x-y|}=\limsup _{\mathcal{S} \ni y \rightarrow x} \frac{|D g(x)(y-x)|}{|x-y|} \\
\leq \limsup _{y \rightarrow x} \frac{\left|D g(x)\left(\pi_{x} y-x\right)\right|}{|x-y|}+\limsup _{y \rightarrow x} \frac{\left|D g(x)\left(\pi_{x} y-y\right)\right|}{|x-y|} .
\end{gathered}
$$

Here

$$
\limsup _{y \rightarrow x} \frac{\left|D g(x)\left(\pi_{x} y-y\right)\right|}{|x-y|} \leq \limsup _{y \rightarrow x} \frac{\left|\pi_{x} y-y\right|}{|x-y|}\|D g(x)\|=0,
$$

by Corollary 2.13. Thus there is actually equality in (7), and hence by the definition of the set $P_{f}$,

$$
\operatorname{Lip} f(x)=\limsup _{y \rightarrow x} \frac{\left|D f(x)\left(\pi_{x} y-x\right)\right|}{|x-y|}=\sup _{y=1}|D f(x) y|=\|D f(x)\| .
$$

This proves (1). Now assume that $f$ is continuous. Then according to Remark 2.7 Lip $f$ is a Borel function. Assume that $U \subset[0, \infty]$ is open. If $\infty \in U$ we have

$$
\begin{aligned}
\|D f(\cdot)\|^{-1}(U) & =\|D f(\cdot)\|^{-1}(U) \cap P_{f} \cup\|D f(\cdot)\|^{-1}(U) \cap P_{f}^{c} \\
& =P_{f} \cup\left((\text { Lip } f)^{-1}(U) \cap P_{f}^{c}\right),
\end{aligned}
$$

which is a Borel set. If $\infty \notin U$ we have

$$
\begin{aligned}
\|D f(\cdot)\|^{-1}(U) & =\|D f(\cdot)\|^{-1}(U) \cap P_{f} \cup\|D f(\cdot)\|^{-1}(U) \cap P_{f}^{c} \\
& =\emptyset \cup(\operatorname{Lip} f)^{-1}(U) \cap P_{f}^{c},
\end{aligned}
$$

which is a Borel set. Thus $\|D f(\cdot)\|$ is a Borel function and (2) is proven. If $f$ is locally Lipschitz it is differentiable almost everywhere; in particular $\mathcal{H}^{n}\left(P_{f}\right)=0$. Thus by (1) and Remark 2.7 there exists constant $C(n)$ such that for almost every $x \in \mathcal{S}$ and for every locally integrable upper gradient $h$ of $f$ we have

$$
\|D f(x)\|=\operatorname{Lip} f(x) \leq C h(x) .
$$

Here $\|D f(x)\|=\operatorname{Lip} f(x)$ for every $x \in \mathcal{S} \backslash P_{f}, \operatorname{Lip} f$ is an upper gradient of $f$ and $\|D f(x)\|=\infty$ for $x \in P_{f}$. Thus we conclude that (3) holds. For (4) it suffices to show that the upper gradient inequality (10) holds for $p$-almost every rectifiable $\gamma$. Since $f \in N^{1, p}(\mathcal{S})$ it has a $p$-integrable $p$-weak upper gradient. Therefore $f \circ \gamma$ is absolutely continuous for $p$-almost every rectifiable path $\gamma$; see 11, Proposition 5.3.3]. Fix such $\gamma:\left[0, l_{\gamma}\right] \rightarrow \mathcal{S}$ parametrized by the arc length. Then

$$
\left|(f \circ \gamma)(0)-(f \circ \gamma)\left(l_{\gamma}\right)\right| \leq \int_{0}^{l_{\gamma}}\left|(f \circ \gamma)^{\prime}(t)\right| d t .
$$


If $f \circ \gamma$ is differentiable at $t$ and $\gamma(t) \in \mathcal{S} \backslash P_{f}$, we have

$$
\left|(f \circ \gamma)^{\prime}(t)\right|=\lim _{s \rightarrow t} \frac{|(f \circ \gamma)(s)-(f \circ \gamma)(t)|}{|\gamma(s)-\gamma(t)|} \leq \operatorname{Lip} f(\gamma(t))=\|D f(\gamma(t))\| .
$$

On the other hand, if $\gamma(t) \in P_{f}$, then $\|D f(\gamma(t))\|=\infty$. This proves the last part of the lemma.

Lemma 2.29. Let $f \in N^{1, n}\left(\mathcal{S}, \mathbb{R}^{k}\right)$ and let $g$ be an $n$-weak locally $n$-integrable upper gradient of $f$. Then

$$
\| \text { apD } f(x) \| \leq C g(x),
$$

for almost every $x \in \mathcal{S}$. Here the constant $C$ depends only on $\mathcal{S}$. In particular, $\|\operatorname{apD} f(x)\| \in L^{n}(\mathcal{S})$.

Proof. Fix $f \in N^{1, n}\left(\mathcal{S}, \mathbb{R}^{k}\right)$ and let sets $E_{i}$ and Lipschitz mappings $f_{i}$ be as in the proof of Corollary 2.17. Then $\mathcal{H}^{n}\left(\bigcap_{i} E_{i}\right)=0$. Furthermore $f$ coincides with $f_{i}$ in $\mathcal{S} \cap E_{i}^{c}$ and for almost every $x \in \mathcal{S} \backslash \bigcap_{i} E_{i}$ there is $i \in \mathbb{N}$ such that

$$
\|\operatorname{apD} f(x)\|=\left\|a p D f_{i}(x)\right\|=\left\|D f_{i}(x)\right\|<\infty .
$$

Let $g$ be an $n$-weak locally $n$-integrable upper gradient of $f$. Because $f$ coincides with $f_{i}$ in $\mathcal{S} \cap E_{i}^{c}$ and because $\mathcal{H}^{n}$ is Borel regular it follows from Lemma 2.8 and Lemma 2.28 that the function $h_{i}: \mathcal{S} \rightarrow[0, \infty]$,

$$
h_{i}(x)=\chi_{E_{i}^{c}}(x) g(x)+\chi_{E_{i}}(x)\left\|D f_{i}(x)\right\|
$$

is an $n$-weak locally $n$-integrable upper gradient of $f_{i}$. Thus by Lemma 2.28 there is constant $C$ such that for every $i$ and for almost every $x \in \mathcal{S}$,

$$
\left\|D f_{i}(x)\right\| \leq C h_{i}(x) \text {. }
$$

We conclude that for almost every $x \in \mathcal{S}$ there is $i \in \mathbb{N}$ such that $x \in E_{i}^{c}$ and

$$
\|a p D f(x)\|=\left\|a p D f_{i}(x)\right\| \leq C h_{i}(x)=C g(x) .
$$

This proves the lemma.

Corollary 2.30. If $\left\{f_{i}\right\}_{i=1}^{\infty}$ is a sequence converging to $f \in N^{1, n}\left(\mathcal{S}, \mathbb{R}^{k}\right)$, then

$$
\left\|a p D\left(f_{i}-f\right)(\cdot)\right\| \rightarrow 0
$$

in $L_{l o c}^{n}(\mathcal{S})$. In particular,

$$
\left\|a p D f_{i}\right\| \rightarrow\|a p D f\|
$$

in $L_{\text {loc }}^{n}(\mathcal{S})$ as $i \rightarrow \infty$.

Proof. If $f_{i} \rightarrow f$ in $N^{1, n}\left(\mathcal{S}, \mathbb{R}^{k}\right)$, we find a sequence $\left\{g_{i}\right\}_{i=1}^{\infty}$ of upper gradients of $f-f_{i}$ such that $g_{i} \rightarrow 0$ in $L^{n}(\mathcal{S})$ as $i \rightarrow \infty$. Now

$$
\left|\|a p D f(x)\|-\left\|a p D f_{i}(x)\right\|\right| \leq\left\|a p D\left(f-f_{i}\right)(x)\right\| \leq C g_{i}(x),
$$

for almost every $x \in \mathcal{S}$. The claim follows.

The following corollary is the main result of this section.

Corollary 2.31. If a sequence $\left\{f_{i}\right\}_{i=1}^{\infty}$ converges to $f$ in $N^{1, n}\left(\mathcal{S}, \mathbb{R}^{n}\right)$, then $\left\{J_{f_{i}}\right\}_{i=1}^{\infty}$ converges to $J_{f}$ in $L^{1}(\mathcal{S})$. 
Proof. Let $f$ and $g$ be two mappings in $N^{1, n}\left(\mathcal{S}, \mathbb{R}^{n}\right)$. Recall that for almost every $x \in \mathcal{S}$ we have $\|\operatorname{apD} f(x)\|=\sup _{|y|=1}|\operatorname{apD} f(x) y|$. Thus it is a linear algebraic fact that for almost every $x \in \mathcal{S}$,

$$
\left|J_{f}(x)-J_{g}(x)\right| \leq \sum_{i=1}^{n}\|\operatorname{apD} f(x)\|^{i-1}\|\operatorname{apD} g(x)\|^{n-i}\|\operatorname{apD} f(x)-\operatorname{apDg}(x)\| .
$$

Therefore, by Hölder's inequality

$$
\begin{aligned}
& \int_{\mathcal{S}}\left|J_{f}(x)-J_{g}(x)\right| d \mathcal{H}^{n}(x) \\
& \leq\left(\int_{\mathcal{S}}\|\operatorname{ap} D f(x)-\operatorname{apD} g(x)\|^{n} d \mathcal{H}^{n}(x)\right)^{\frac{1}{n}} \\
& \times \sum_{i=1}^{n}\left(\int_{\mathcal{S}}\|a p D f(x)\|^{\frac{n}{n-1}(i-1)}\|a p D g(x)\|^{\frac{n}{n-1}(n-i)} d \mathcal{H}^{n}(x)\right)^{\frac{n-1}{n}} .
\end{aligned}
$$

Here, for every $i \in\{2, \ldots, n-1\}$ we have

$$
\begin{aligned}
& \int_{\mathcal{S}}\|\operatorname{apD} f(x)\|^{\frac{n}{n-1}(i-1)}\|\operatorname{apDg}(x)\|^{\frac{n}{n-1}(n-i)} d \mathcal{H}^{n}(x) \\
& \leq\left(\int_{\mathcal{S}}\|a p D f(x)\|^{n} d \mathcal{H}^{n}(x)\right)^{\frac{n-1}{n-i}}\left(\int_{\mathcal{S}}\|a p D g(x)\|^{n} d \mathcal{H}^{n}(x)\right)^{\frac{n-1}{i-1}} .
\end{aligned}
$$

Hence

$$
\begin{aligned}
& \int_{\mathcal{S}}\left|J_{f}(x)-J_{g}(x)\right| d \mathcal{H}^{n}(x) \\
& \leq\left(\int_{\mathcal{S}}\|\operatorname{apD} f(x)-\operatorname{apD} D(x)\|^{n} d \mathcal{H}^{n}(x)\right)^{\frac{1}{n}} \\
& \times\left(\int_{\mathcal{S}}\|\operatorname{apD} D(x)\|^{n}+\|a p D f(x)\|^{n} d \mathcal{H}^{n}(x)\right. \\
& \left.+\sum_{i=2}^{n-1}\left(\int_{\mathcal{S}}\|a p D f(x)\|^{n} d \mathcal{H}^{n}(x)\right)^{\frac{n-1}{n-i}}\left(\int_{\mathcal{S}}\|a p D g(x)\|^{n} d \mathcal{H}^{n}(x)\right)^{\frac{n-1}{i-1}}\right)^{\frac{n-1}{n}} .
\end{aligned}
$$

According to Corollary 2.30, $f_{i} \rightarrow f$ implies $\left\|a p D f_{i}(\cdot)-a p D f(\cdot)\right\| \rightarrow 0$ in $L^{n}(\mathcal{S})$. Thus the last inequality gives the claim.

2.4. Generalized manifolds. In this section we discuss topological assumptions required on the space $\mathcal{S}$. We follow [12] also see [13. We assume that $\mathcal{S}$ is locally compact, separable, connected, and locally connected. Let $H_{c}^{*}(\mathcal{S})$ denote the Alexander-Spanier cohomology groups of $\mathcal{S}$, with compact supports and coefficients in $\mathbb{Z}$. We assume that $\mathcal{S}$ is a cohomology $n$-manifold. That is:

(1) The topological dimension of $\mathcal{S}$ is at most $n$.

(2) The local cohomology groups of $\mathcal{S}$ are equivalent to $\mathbb{Z}$ in degree $n$ and equivalent to 0 in degree $n-1$.

The last condition means that for every point $x \in \mathcal{S}$ and for every open neighborhood $U$ of $x$, there is another open neighborhood $V$ of $x$ contained in $U$, such that

$$
H_{c}^{p}(V)= \begin{cases}\mathbb{Z} & \text { if } p=n \\ 0 & \text { if } p=n-1\end{cases}
$$


The standard homomorphism

$$
H_{c}^{n}(W) \rightarrow H_{c}^{n}(V)
$$

is a surjection whenever $W$ is open neighborhood of $x$ included in $V$. We assume that $H_{c}^{n}(\mathcal{S}) \approx \mathbb{Z}$. That is, $\mathcal{S}$ is orientable, and a generator $g_{\mathcal{S}}$ is called an orientation of $\mathcal{S}$. The pair $\left(\mathcal{S}, g_{\mathcal{S}}\right)$ is called an oriented generalized $n$-manifold. Assuming $\mathcal{S}$ is oriented, we can choose a coherent orientation to every connected open subset $U$ of $\mathcal{S}$, via the isomorphism

$$
H_{c}^{n}(U) \rightarrow H_{c}^{n}(\mathcal{S})
$$

Now we are ready to define local degree. We say that $D \subset \mathcal{S}$ is a domain if it is open and connected. A set $D \subset \mathcal{S}$ is called relatively compact if the closure $\bar{D}$ is compact. Let $\mathcal{S}$ and $Y$ be two oriented generalized $n$-manifolds, and $f: \mathcal{S} \rightarrow Y$ continuous. For every relatively compact domain $D$ and for each component $V$ of $Y \backslash f(\partial D)$, the map

$$
f_{\mid f^{-1}(V) \cap D}: f^{-1}(V) \cap D \rightarrow V
$$

is proper. That is, the preimage of every compact set is compact. Hence we have the following sequence of maps

$$
H_{c}^{n}(V) \rightarrow H_{c}^{n}\left(f^{-1}(V) \cap D\right) \rightarrow H_{c}^{n}(D) .
$$

Here the first map is induced by $f$ and the second one is the isomorphism provided by assumption (8). The composed map sends the generator of $H_{c}^{n}(V)$ to some integer multiple of the generator of $H_{c}^{n}(D)$. This integer is denoted by $\mu(y, D, f)$ and called the local degree of mapping $f$, at point $y \in V$, with respect to $D$. Note that the value of the function $y \mapsto \mu(y, f, D)$ does not depend on point $y$, but on the component of $f(\partial D)^{C}$. Thus the function is constant on every component of $f(\partial D)^{C}$. Note also that $\mu$ is defined only in $f(\partial D)^{C}$.

Definition 2.32. A continuous map $f: \mathcal{S} \rightarrow Y$ is sense-preserving if $\mu(y, f, D)>0$ for every relatively compact domain $D$ in $\mathcal{S}$ and for every point $y \in f(D) \backslash f(\partial D)$.

Using the definition and basic properties of Alexander-Spanier cohomology it is not too difficult to see that topological degree enjoys the properties listed in Lemma 2.33

Lemma 2.33. Let $U \subset \mathcal{S}$ be a domain. Topological degree satisfies the following:

(1) If $\left\{U_{1}, U_{2}, \ldots U_{k}\right\}$ is a collection of domains with $U_{i} \cap U_{j}=\emptyset$, for every $i \neq j$ and $U \cap f^{-1}(y) \subset \bigcup_{i=1}^{k} U_{i} \subset U$, then

$$
\mu(y, f, U)=\sum_{i=1}^{k} \mu\left(y, f, U_{i}\right),
$$

for every $y \in f(U) \backslash f(\partial U)$.

(2) Let $f$ and $g$ be two mappings from $\mathcal{S}$ to $\mathbb{R}^{n}$. Let $y \in \mathcal{S}$ be a point such that there is homotopy $h_{t}$ between $f$ and $g$ with $y \notin h_{t}(\partial U)$ for every $t$. Then $\mu(y, f, U)=\mu(y, g, U)$.

(3) Let $T: \mathbb{R}^{n} \rightarrow \mathbb{R}^{n}$ be a linear bijection. Then $\mu(y, T, U)=\operatorname{sgn}(\operatorname{det} T)$, for every $y \in T(U)$. 
Next we consider the relation between the orientation of approximate tangent planes of an $n$-rectifiable set $\mathcal{S} \subset \mathbb{R}^{m}$ and the orientation of $\mathcal{S}$. Let $U$ be an open subset of $\mathcal{S}$. According to Theorem 2.12, for almost every $x \in \mathcal{S}$ there is a unique $n$ dimensional plane $\operatorname{ap} \operatorname{Tan}(x, \mathcal{S})$. Denote the collection of all these planes associated to points in $U$ by $T U$. A measurable choice of orientation $g_{x}$ on each $\operatorname{apTan}(x, U)$ in $T U$ is called an orientation of the tangent bundle $T U$. According to [5, Lemma 3.2.25] such orientation above always exists. Because $\mathcal{S}$ is an oriented, generalized $n$-manifold, there is a fixed orientation of $U$ induced by the mapping in (8), provided that $U$ is connected. Fix $x \in U$, such that $\operatorname{ap} \operatorname{Tan}(x, U) \in T U$ exists. According to Remark 2.14 the projection $\pi_{x}: \mathbb{R}^{m} \rightarrow \operatorname{apTan}(x, U)$ satisfies $x \notin \pi(\partial D)$ when $D$ is a small open connected neighborhood of $x$ in $U$. Thus, if $V$ is the $x$-component of $\operatorname{apTan}(x, U) \backslash \pi_{x}(\partial D)$, we have

$$
H_{c}^{n}(\operatorname{apTan}(x, U)) \leftarrow H_{c}^{n}(V) \stackrel{\pi_{x}^{*}}{\longrightarrow} H_{c}^{n}\left(\pi_{x}^{-1}(V) \cap D\right) \rightarrow H_{c}^{n}(D) \rightarrow H_{c}^{n}(U) .
$$

Here $\pi_{x}^{*}$ denotes the homomorphism induced by $\pi_{x}$, and unnamed arrows represent canonical isomorphisms induced by embeddings. We say that $U$ is metrically orientable if there is an orientation of tangent bundle $T U$ such that for almost every $x \in U g_{x}$ is mapped to $g_{U}$ under the mapping represented above. If such an orientation of $T U$ is chosen we say that $U$ is metrically oriented. Finally, we say that $\mathcal{S}$ is locally metrically orientable if every point in $\mathcal{S}$ has a neighborhood that is metrically orientable. Note that to say that $U$ is metrically oriented is to say that $U$ is oriented via the isomorphism in (8) and $\mu\left(y, \pi_{x}, U\right)=1$, whenever defined. From now on, we assume that the space $\mathcal{S}$ is locally metrically orientable and orientations as described above are chosen. Note that this fixes an orthonormal basis for each tangent plane $\operatorname{ap} \operatorname{Tan}(x, \mathcal{S})$. In particular Definition 2.22 is now justified.

Definition 2.34. Let $X$ be a metric space. We say that $X$ is linearly locally contractible if for every compact set $K \subset X$ there exist numbers $r_{K}>0$ and $C_{K} \geq 1$ such that for every $x \in K$ and every $r \leq r_{K}$ the ball $B(x, r) \subset X$ contracts to a point inside $B\left(x, C_{K} r\right)$. This means that there exists a continuous map $h: B(x, r) \times[0,1] \rightarrow B\left(x, C_{K} r\right)$ such that $h(\cdot, 0): B(x, r) \rightarrow B\left(x, C_{K} r\right)$ is the identity map and $h(\cdot, 1): B(x, r) \rightarrow B\left(x, C_{K} r\right)$ is a constant map $h(\cdot, 1)=y$, for some $y \in B\left(x, C_{K} r\right)$.

Remark 2.35. If we assume that $\mathcal{S}$ is linearly locally contractible, then $\mathcal{S}$ is locally metrically orientable; see [13, Example 3.10]. Actually, it follows from work of Semmes 24] that if a metric space $X \subset \mathbb{R}^{m}$ is Ahlfors $n$-regular, has integral cohomology modules as described above and is also linearly locally contractible, then $X$ supports a weak 1-Poincaré inequality. Furthermore, by [4, Theorem 14.2] this implies that $X$ is actually $n$-rectifiable. Thus on this paper one can assume that $\mathcal{S} \subset \mathbb{R}^{m}$ is an Ahlfors $n$-regular, linearly locally contractible cohomology $n$ manifold. This implies that $\mathcal{S}$ is $n$-rectifiable, metrically orientable and supports a weak 1-Poincaré inequality, and thus this is an example of a setting where our definition of mapping of finite distortion makes sense and our main theorems, Theorem 3.2 and Theorem 3.3 apply.

2.5. Path lifting for open and discrete maps. In this section we consider some properties of continuous, discrete and open mappings between two generalized manifolds. Such mappings are called branched covers. It is proved by Väisälä in 27] that a branched cover between generalized manifolds is either sense-preserving 
or sense-reserving. In this section we always assume that mapping denoted by $f$ is a branched cover.

Definition 2.36. Let $X$ and $Y$ be two generalized manifolds. For every $x \in X$ there is a relatively compact neighborhood $D$ such that $f^{-1}(f(x)) \cap \bar{D}=x$. The number $\mu(f(x), D, f)$ is called the local index of $f$ at $x$ and denoted by $i(x, f)$. The local index is independent of choice of $D$.

Next we describe the path lifting property for a sense-preserving branched cover $f$ between generalized manifolds $X$ and $Y$. Let $\beta:[a, b[\rightarrow Y$ be a path. A maximal $f$-lifting of $\beta$ starting at point $x \in f^{-1}(f(a))$ is a path $\alpha:[a, c[\rightarrow X, c \leq b$ such that $\alpha(a)=x, f \circ \alpha=\beta_{\mid[a, c \mid}$ and that if $c^{\prime}>c$ then there is no path $\alpha^{\prime}:\left[a, c^{\prime}[\rightarrow Y\right.$

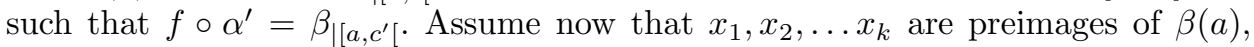
then there exists a maximal sequence $\alpha_{1}, \alpha_{2}, \ldots, \alpha_{m}$ of $f$-liftings of $\beta$ starting at points $x_{1}, x_{2}, \ldots x_{k}$. This means that each $\alpha_{j}:\left[a, c_{j}[\rightarrow X\right.$ is a maximal $f$-lifting of $\beta$ starting at $x_{i}$ for some $i=1,2, \ldots, k$ and

$$
m=\sum_{i=1}^{k} i\left(x_{i}, f\right) .
$$

Furthermore it is required that

$$
\operatorname{card}\left\{j: \alpha_{j}(a)\right\}=i\left(x_{i}, f\right)
$$

and

$$
\text { card }\left\{j: \alpha_{j}(s)=x\right\} \leq i(x, f),
$$

for every $s \in\left[a, c_{j}\right.$ [ and every $x \in X$. The existence of maximal $f$-liftings can be proved as in 23 .

\section{Mappings of Finite Distortion}

Now we are ready to give a definition for mappings of finite distortion on $n$ rectifiable, Ahlfors $n$-regular set $\mathcal{S} \subset \mathbb{R}^{m}, m \geq n$ that is metrically oriented cohomology $n$-manifold, supporting a weak $n$-Poincaré inequality.

Definition 3.1. We say that a continuous $f \in N_{l o c}^{1, n}\left(\mathcal{S}, \mathbb{R}^{n}\right)$ is a mapping of finite distortion if there is a measurable function $K: \mathcal{S} \rightarrow[1, \infty$ [ satisfying

$$
\|\operatorname{apD} f(x)\|^{n} \leq K(x) J_{f}(x)
$$

for almost every $x \in \mathcal{S}$.

We will prove the following theorems.

Theorem 3.2. Let $f: \mathcal{S} \rightarrow \mathbb{R}^{n}$ be a mapping of finite distortion. Then $f$ is sensepreserving, monotone, differentiable $\mathcal{H}^{n}$-almost everywhere and it maps sets of $\mathcal{H}^{n}$ measure zero to sets of $\mathcal{H}^{n}$-measure zero.

Theorem 3.3. Let $f: \mathcal{S} \rightarrow \mathbb{R}^{n}$ be a mapping of finite distortion with $K \in L_{\text {loc }}^{p}(\mathcal{S})$, for some $p>n-1$, and let $\mathcal{S}$ support a weak $(n-1)$-Poincaré inequality. If $f$ is nonconstant, then $f$ is open and discrete.

Remark 3.4. It follows from Definition 3.1 and from Lemma 2.29 that $J_{f} \in L_{l o c}^{1}(\mathcal{S})$. In Definition 3.1 we assumed that a mapping of finite distortion is continuous. In the Euclidean case a mapping of finite distortion of class $W_{l o c}^{1, n}\left(\Omega, \mathbb{R}^{n}\right)$ is always continuous. Actually, in the Euclidean case one defines mappings of finite distortion 
by asking $f$ to satisfy the distortion inequality (12) and to belong to Sobolev space $W_{l o c}^{1,1}\left(\Omega, \mathbb{R}^{n}\right)$, with $J_{f} \in L_{\text {loc }}^{1}(\Omega)$ but we need to assume that $f$ belong to $N_{l o c}^{1, n}\left(\mathcal{S}, \mathbb{R}^{n}\right)$ since otherwise we cannot guarantee that $f$ has approximate derivative and thus the distortion inequality would not necessarily make sense. However, if one assumes that $\mathcal{S}$ supports a weak 1-Poincaré inequality, then approximate derivative exists if $f$ is only assumed to belong to the Newtonian space $N_{l o c}^{1,1}\left(\mathcal{S}, \mathbb{R}^{n}\right)$. Recall from Remark 2.35 that if $\mathcal{S}$ is locally linearly contractible, it will support a 1-Poincaré inequality.

\section{Discrete COnVOlution}

In this section we assume that $X$ is an Ahlfors $n$-regular space supporting a weak $n$-Poincaré inequality. We introduce the so-called discrete convolution for Newtonian functions. This gives a tool to approximate Newtonian mappings by Lipschitz mappings. To be more precise, if we assume that $f \in N_{l o c}^{1, n}(X)$ is continuous, then discrete convolution gives a sequence of locally Lipschitz mappings which converges to $f$ in $N_{l o c}^{1, n}(X)$ and uniformly on compact sets. We need this property for the sequence in order to show that a continuous Newtonian mapping $f$ with $J_{f}(x) \geq 0$ a.e. is sense-preserving. In this section $C$ denotes a generic positive constant depending only on the Ahlfors regularity constant $C_{A}$, on the dimension $n$ and on the constants on the Poincaré inequality. The value of $C$ may vary from line to line. Our main references in this section are [1] and [16].

The space $X$ can be covered by balls as follows. Fix $r>0$, and to every point $x \in X$ associate a ball $B(x, r)$. By the Vitali covering theorem, one can find a subcollection of $\left\{B_{i}\right\}_{i=1}^{\infty}$, such that the balls $B_{i}$ and $B_{j}$ are disjoint whenever $i \neq j$ but the space $X$ is covered by the balls $\left\{6 B_{i}\right\}_{i=1}^{\infty}$. Since $X$ is Ahlfors regular and the balls $\left\{B_{i}\right\}_{i=1}^{\infty}$ are disjoint, it is not hard to see that if $\bigcap_{i=1}^{p} 6 B_{i} \neq \emptyset$, then $p$ is bounded above by a constant $C$ not depending on $r$. For every $i \in \mathbb{N}$, set

$$
\phi_{i}(x)= \begin{cases}1 & x \in B\left(x_{i}, 3 r\right), \\ 2-\frac{\left|x_{i}-x\right|}{3 r} & x \in B\left(x_{i}, 6 r\right) \backslash B\left(x_{i}, 3 r\right), \\ 0 & \text { otherwise. }\end{cases}
$$

Finally, we set

$$
\Psi_{i}(x)=\frac{\phi_{i}(x)}{\sum_{j=1}^{\infty} \phi_{j}(x)} .
$$

Then $\sum_{i=1}^{\infty} \Psi_{i}(x)=1$ for every $x \in X$. Note that for each $x \in X$, the sum is finite since the overlap of the balls $6 B_{i}$ is bounded. Furthermore, $\Psi_{i}$ is $\frac{C}{r}$-Lipschitz.

Definition 4.1. Let $f \in L_{l o c}^{1}(X)$. Fix $r>0$ and balls $B_{i}$ as above. We define the discrete convolution of $f$ as

$$
f_{r}(x)=\sum_{i=1}^{\infty} f_{3 B_{i}} \Psi_{i}(x),
$$

here $f_{3 B_{i}}=f_{3 B_{i}} f(x) d \mathcal{H}^{n}(x)$.

Lemma 4.2. Let $f \in L_{l o c}^{1}(X)$ and $r>0$. Then $f_{r}$ is locally Lipschitz. In addition, for any $n$-weak $n$-integrable upper gradient $g$ of $f$, and for any $z \in X$ and $R>106 \lambda r$ we have

$$
\int_{B(z, R)} \operatorname{Lip}_{r}(x)^{n} d \mathcal{H}^{n}(x) \leq C \int_{B(z, 2 R)} g(x)^{n} d \mathcal{H}^{n}(x) .
$$


Proof. Fix $x \in X$ and $r>0$. Let $B_{i_{0}}$ be a ball as in the definition of $f_{r}$, with $x \in 6 B_{i_{0}}$. For any $y, z \in 6 B_{i_{0}}$ we have

$$
\begin{aligned}
\left|f_{r}(y)-f_{r}(z)\right| & =\left|\sum_{i=1}^{\infty} f_{3 B_{i}} \Psi_{i}(y)-\sum_{i=1}^{\infty} f_{3 B_{i}} \Psi_{i}(z)\right| \\
& =\left|\sum_{i=1}^{\infty} f_{3 B_{i}} \Psi_{i}(y)-\sum_{i=1}^{\infty} f_{3 B_{i}} \Psi_{i}(z)+\sum_{i=1}^{\infty} f_{3 B_{i_{0}}} \Psi_{i}(z)-\sum_{i=1}^{\infty} f_{3 B_{i_{0}}} \Psi_{i}(y)\right| \\
& =\left|\sum_{i=1}^{\infty}\left(f_{3 B_{i}}-f_{3 B_{i_{0}}}\right) \Psi_{i}(y)-\sum_{i=1}^{\infty}\left(f_{3 B_{i}}-f_{3 B_{i_{0}}}\right) \Psi_{i}(z)\right| \\
& =\left|\sum_{i=1}^{\infty}\left(f_{3 B_{i}}-f_{3 B_{i_{0}}}\right)\left(\Psi_{i}(y)-\Psi_{i}(z)\right)\right| \\
& \leq C \max _{6 B_{i} \cap 6 B_{i_{0}} \neq \emptyset}\left|f_{3 B_{i}}-f_{3 B_{i_{0}}}\right| \frac{1}{r}|y-z| .
\end{aligned}
$$

We conclude that $f_{r}$ is locally Lipschitz. Let $g$ be an $n$-weak $n$-integrable upper gradient of $f$. Then by the Poincaré inequality, for every $B_{i}$ with $6 B_{i} \cap 6 B_{i_{0}} \neq \emptyset$, we have

$$
\begin{aligned}
\left|f_{3 B_{i}}-f_{3 B_{i_{0}}}\right| & \leq\left|f_{3 B_{i}}-f_{B(x, 100 r)}\right|+\left|f_{3 B_{i_{0}}}-f_{B(x, 100 r)}\right| \\
& \leq C f_{B(x, 100 r)}\left|f_{B(x, 100 r)}-f(y)\right| d \mathcal{H}^{n}(y) \\
& \leq C r\left(f_{B(x, \lambda 100 r)} g(y)^{n} d \mathcal{H}^{n}(y)\right)^{\frac{1}{n}} .
\end{aligned}
$$

This together with (13) gives

$$
\operatorname{Lip}_{r}(x) \leq C\left(f_{B(x, \lambda 100 r)} g(y)^{n} d \mathcal{H}^{n}(y)\right)^{\frac{1}{n}}
$$

for every $x \in X$. Now fix $z \in X$ and $R>\lambda 106 r$. By the Vitali covering theorem, the ball $B(z, R)$ can be covered by balls $\left\{B\left(x_{i}, 6 r\right)\right\}_{i=1}^{\infty}$ so that $x_{i} \in B(z, R)$ for every $i$, and $B\left(x_{i}, r\right) \cap B\left(x_{j}, r\right)=\emptyset$, whenever $i \neq j$. As mentioned earlier, the Ahlfors regularity implies that the overlap of the balls $B\left(x_{i}, \lambda 106 r\right)$ is bounded by a constant depending only on the Ahlfors constant $C_{A}$, and on dimension $n$ but not on radius $r$. Using such a cover $\left\{B\left(x_{i}, 6 r\right)\right\}_{i=1}^{\infty}$, we get

$$
\begin{aligned}
& \int_{B(z, R)} \operatorname{Lip} f_{r}(x)^{n} d \mathcal{H}^{n}(x) \leq \sum_{i} \int_{B\left(x_{i}, 6 r\right)} \frac{C}{r^{n}} \int_{B(x, \lambda 100 r)} g(y)^{n} d \mathcal{H}^{n}(y) d \mathcal{H}^{n}(x) \\
& \leq \sum_{i} \int_{B\left(x_{i}, 6 r\right)} \frac{C}{r^{n}} \int_{B\left(x_{i}, \lambda 106 r\right)} g(y)^{n} d \mathcal{H}^{n}(y) d \mathcal{H}^{n}(x) \\
& \leq C \sum_{i} \int_{B\left(x_{i}, \lambda 106 r\right)} g(y)^{n} d \mathcal{H}^{n}(y) \leq C \int_{B(z, 2 R)} g(y)^{n} d \mathcal{H}^{n}(y) .
\end{aligned}
$$

This proves the claim.

Lemma 4.3. Let $f \in L_{\text {loc }}^{1}(X)$. Then $f_{r} \rightarrow f$ pointwise almost everywhere in $X$ as $r \rightarrow 0$. In particular, if $f$ is continuous, then $f_{r} \rightarrow f$ uniformly on compact sets. 
Proof. Fix $x \in X$ and pick a ball $B_{i_{0}}$ as in the definition of $f_{r}$ such that $x \in 6 B_{i_{0}}$. Then

$$
\begin{aligned}
\left|f_{r}(x)-f(x)\right|= & \left|\sum_{i=1}^{\infty} f_{3 B_{i}} \Psi_{i}(x)-\sum_{i=1}^{\infty} f(x) \Psi_{i}(x)\right| \\
& \leq \sum_{i=1}^{\infty}\left|f_{3 B_{i}}-f(x)\right| \Psi_{i}(x) \leq \sum_{i=1}^{\infty} f_{3 B_{i}}|f(x)-f(y)| d \mathcal{H}^{n}(y) \Psi_{i}(x) \\
& \leq C f_{100 B_{i_{0}}}|f(y)-f(x)| d \mathcal{H}^{n}(y) .
\end{aligned}
$$

Because $f \in L_{l o c}^{1}(X)$, for almost every $x \in X$ the last integral of (14) tends to zero, as $r$ tends to zero. Furthermore, if $f$ is continuous it is uniformly continuous on compact sets. This together with (14) gives the last part of the claim.

Remark 4.4. Suppose that $K \subset X$ is compact and $f \in N_{l o c}^{1, n}(X)$ is a continuous function. Let $\left\{f_{i}\right\}_{i=1}^{\infty}$ be a sequence of discrete convolutions of $f$ such that $r_{i} \rightarrow 0$ as $i \rightarrow \infty$. Since $f_{i}$ is locally Lipschitz, it has upper gradient Lip $f_{i}$. Furthermore, by Lemma 4.2 the sequence $\left\{\operatorname{Lip} f_{i}\right\}_{i=1}^{\infty}$ is bounded on $L^{n}(K)$. Thus, by Mazur's lemma there is the function $f_{0} \in N^{1, n}(K)$ and a sequence of convex combinations

$$
f_{k}^{*}=\sum_{i=1}^{m_{k}} a_{k_{i}} f_{i},
$$

converging to $f_{0}$ in $N_{l o c}^{1, n}(K)$. Here the coefficients satisfy $a_{k_{i}} \geq 0$ and $\sum_{i=1}^{m_{k}} a_{k_{i}}=1$, for every $k$. It is easy to see that the sequence of convex combinations converges to $f$ uniformly on compact sets. Thus $\left\|f-f_{0}\right\|_{L^{n}(K)}=0$. By [25, Corollary 3.3] we conclude that $f_{0}=f$ also in Newtonian sense. Note that every element of the sequence of convex combination is locally Lipschitz. Thus we have found a way to approximate Newtonian functions by locally Lipschitz functions such that the convergence is uniform on compact sets. If $f$ maps to $\mathbb{R}^{k}$, we can apply our result to every component function and the properties of the approximate sequence will be preserved.

From now on, we assume that $\mathcal{S} \subset \mathbb{R}^{m}$ is Ahlfors $n$-regular metrically oriented cohomology $n$-manifold, supporting a weak $n$-Poincaré inequality. We write $C(\mathcal{S})$ for any positive constant depending only on constants associated to space $\mathcal{S}$. The value of $C(\mathcal{S})$ may vary from line to line.

\section{Non-NEGAtive JaCOBIAN AND SEnSE-PRESERVING MAPS}

Now we have introduced all the machinery needed in order to show that a continuous Newtonian mapping with almost everywhere non-negative Jacobian determinant is sense-preserving. This is Theorem 5.3 which is the main result of this section.

Lemma 5.1. Let $f: \mathcal{S} \rightarrow \mathbb{R}^{n}$ be a locally Lipschitz map and $D \subset \mathcal{S}$ a relatively compact domain. Let $y \in f(D) \backslash f(\partial D)$, such that $f$ is differentiable at $x$ and $J_{f}(x)>0$ for every $x \in f^{-1}(y) \cap D$. In addition assume that card $f^{-1}(y) \cap D$ is finite. Then $\mu(y, f, D)>0$.

We postpone the proof of Lemma 5.1 until the end of this section. 
Lemma 5.2. Let $f \in N_{l o c}^{1, n}\left(\mathcal{S}, \mathbb{R}^{n}\right)$ be a continuous map with $J_{f}(x) \geq 0$ for almost every $x \in \mathcal{S}$, and $\left\{f_{i}\right\}_{i=1}^{\infty}$ a sequence of locally Lipschitz maps such that $f_{i} \rightarrow f$ in $N_{\text {loc }}^{1, n}\left(\mathcal{S}, \mathbb{R}^{n}\right)$. Let $D$ be a compact subset of $\mathcal{S}$. Denote $A_{i}=\left\{x \in D: J_{f_{i}}(x) \leq 0\right\}$. Then $\mathcal{H}^{n}\left(f_{i}\left(A_{i}\right)\right) \rightarrow 0$, as $i \rightarrow 0$.

Proof. By Theorem 2.23 and Corollary 2.31, we get

$$
\begin{aligned}
\mathcal{H}^{n}\left(f_{i}\left(A_{i}\right)\right) & =\int_{f_{i}\left(A_{i}\right)} d \mathcal{H}^{n}(y) \leq \int_{f_{i}\left(A_{i}\right)} \sum_{x \in f^{-1}(y)} \chi_{A_{i}}(x) d \mathcal{H}^{n}(y) \\
& =\int_{\mathcal{S}}\left|J_{f_{i}}(x)\right| \chi_{A_{i}}(x) d \mathcal{H}^{n}(x)=\int_{A_{i}}-J_{f_{i}}(x) d \mathcal{H}^{n}(x) \\
& \leq \int_{A_{i}} J_{f}(x)-J_{f_{i}}(x) d \mathcal{H}^{n}(x) \leq \int_{D}\left|J_{f}(x)-J_{f_{i}}(x)\right| d \mathcal{H}^{n}(x) \rightarrow 0,
\end{aligned}
$$

as $i \rightarrow \infty$.

Theorem 5.3. Let $f \in N_{\text {loc }}^{1, n}\left(\mathcal{S}, \mathbb{R}^{n}\right)$ be a continuous map with $J_{f}(x) \geq 0$ for almost every $x \in \mathcal{S}$. Then $f$ is sense-preserving.

Proof. Let $D \subset \mathcal{S}$ be a relatively compact domain. Let $y \in f(D) \backslash f(\partial D)$. We need to show that $\mu(y, f, D)>0$. Let $\left\{f_{i}\right\}_{i=1}^{\infty}$ be a sequence of Lipschitz mappings, converging to $f$ in $N^{1, n}\left(D, \mathbb{R}^{n}\right)$ and uniformly on $D$. Let $Z$ be the $y$-component of $\mathbb{R}^{n} \backslash f(\partial D)$. Since $Z$ is open, we find a ball $B(y, r) \subset Z$. Because $f_{i} \rightarrow f$ uniformly, $f_{i}(\partial D) \cap B(y, r)=\emptyset$ for sufficiently large $i$ and thus $\mu\left(\omega, f_{i}, D\right)$ is well defined whenever $\omega \in B(y, r)$. According to Lemma 5.2, the measure of the set

$$
J_{i}^{+}=\left\{\omega \in B(y, r): J_{f_{i}}(x)>0, \text { for every } x \in f_{i}^{-1}(\omega)\right\}
$$

is positive for large indices $i$. Thus by Theorem 2.23 for sufficiently large $i$ we find $y_{i} \in J_{i}^{+}$such that card $f_{i}^{-1}\left(y_{i}\right) \cap D$ is finite and $f_{i}$ is differentiable at each point of $f_{i}^{-1}\left(y_{i}\right)$. Then, by Lemma $5.1 \mu\left(y_{i}, f_{i}, D\right)>0$. Because $f_{i} \rightarrow f$ uniformly, for sufficiently large indices $i$ there is a homotopy between $f_{i}$ and $f$ as described in Lemma 2.33 and thus for sufficiently large $i$ we have $\mu\left(y_{i}, f_{i}, D\right)=\mu\left(y_{i}, f, D\right)$. On the other hand, topological degree is constant on each component of $\mathbb{R}^{n} \backslash f(\partial D)$. That is,

$$
0<\mu\left(y_{i}, f_{i}, D\right)=\mu\left(y_{i}, f, D\right)=\mu(y, f, D) .
$$

Finally, we give a proof for Lemma 5.1 .

Proof. Fix a relatively compact domain $D \subset \mathcal{S}$ and $y \in f(D) \backslash f(\partial D)$ as in the lemma. Let $\left\{x_{1}, x_{2}, \ldots x_{k}\right\}=f^{-1}(y) \cap D$. Since $f^{-1}(y) \cap D$ is discrete we find balls $B\left(x_{i}, r\right) \subset D$ with pairwise empty intersection. Write $B_{i}$ for the $x_{i}$-component of $B\left(x_{i}, r\right)$. By Lemma 2.33

$$
\mu(y, f, D)=\sum_{i=1}^{k} \mu\left(y, f, B_{i}\right) .
$$

Thus it is sufficient to show that $\mu\left(y, f, B_{i}\right)>0$, for every $i$. Fix $i$. We may assume that $x_{i}=0$ and $y=0$. Since $\mathcal{S}$ is metrically oriented we may assume that $r$ is so small that $\mu\left(0, \pi_{0}, B_{i}\right)=1$. On the other hand, because $J_{f}(0)>0$, it follows from Lemma 2.33 that $\mu\left(0, D f(0), \pi_{0} B_{i}\right)=1$. Hence

$$
\mu\left(0, D f(0) \pi_{0}, B_{i}\right)=1 .
$$


Thus in order to show that $\mu\left(0, f, B_{i}\right)>0$ it is sufficient to show that the homotopy $g_{t}: D \rightarrow \mathbb{R}^{n}$,

$$
g_{t}(z)=t D f(0) \pi_{0}(z)+(1-t) f(z)
$$

has the property $g_{t}(\partial B(0, r)) \cap\{0\}=\emptyset$, for sufficiently small $r>0$. Note that $\partial B_{i} \subset \partial B(0, r)$. Since $J_{f}(0)>0$ we have

$$
0<\lambda:=\inf _{|z|=1}|D f(0) z| .
$$

By Corollary 2.13, $2\left|\pi_{0} z\right| \geq|z|$ if $z \in \mathcal{S}$ and $|z|$ is sufficiently small. Thus

$$
\left|D f(0) \pi_{0} z\right| \geq \frac{\lambda}{2}|z| \text {. }
$$

Because $f$ is differentiable at 0 and we find $g: \mathbb{R}^{m} \rightarrow \mathbb{R}^{n}$ which is differentiable at 0 and coincides with $f$ on $\mathcal{S}$. Therefore

$$
\begin{aligned}
\left|D f(0) \pi_{0} z-f(z)\right| & \leq\left|D g(0) \pi_{0} z-f(z)+D g(0) z-D g(0) z\right| \\
& \leq \frac{\lambda}{16}|z|+\|D g(0)\|\left|\pi_{0} z-z\right| \leq \frac{\lambda}{8}|z|,
\end{aligned}
$$

whenever $z \in \mathcal{S}$ and $|z|$ is sufficiently small. Fix small $r>0$ and let $z \in \partial B(0, r)$. Then

$$
\left|D f(0) \pi_{0} z-g_{t}(z)\right| \leq 2\left|D f(0) \pi_{0} z-f(z)\right| \leq \frac{\lambda}{4}|z| .
$$

This together with (15) implies that $g_{t}(\partial B(0, r)) \cap\{0\}=\emptyset$ for sufficiently small $r$ and for every $t$.

\section{Differentiability and the Lusin COndition}

In this section we prove that a mapping of finite distortion $f$ is differentiable almost everywhere and satisfies the Lusin condition. This means that $f$ maps sets of measure zero to sets of measure zero. In order to prove this we need the following simple lemma which says that $f$ is monotone.

Lemma 6.1. Let $f: \mathcal{S} \rightarrow \mathbb{R}^{n}$ be a continuous sense-preserving map and let $B(x, r)$ be a ball in $\mathcal{S}$. Then

$$
\operatorname{diam} f(\bar{B}(x, r)) \leq \operatorname{diam} f(\partial B(x, r)) .
$$

Proof. Since $f(\bar{B}(x, r))$ is compact, there are points $y, z \in \bar{B}(x, r)$ such that

$$
\operatorname{diam} f(\bar{B}(x, r))=|f(y)-f(z)| .
$$

If $f(y), f(z) \in f(\partial B(x, r))$ the claim follows. Assume that $f(z) \notin f(\partial B(x, r))$. Then $z \in B(x, r)$. Write $B$ for the $z$-component of $B(x, r)$. Then $f(z) \notin f(\partial B) \subset$ $f(\partial B(x, r))$. Since $f$ is sense-preserving, $\mu(f(z), f, B)>0$. On the other hand, topological degree is constant on every component of $\mathbb{R}^{n} \backslash f(\partial B)$ and the components are open. This means that there is a radius $r^{\prime}>0$ such that the ball $B\left(f(z), r^{\prime}\right)$ is contained in the $f(z)$ component of $\mathbb{R}^{n} \backslash f(\partial B)$ and moreover $\mu(\omega, f, B)=$ $\mu(f(z), f, B)>0$, for every $\omega \in B\left(f(z), r^{\prime}\right)$. Thus $B\left(f(z), r^{\prime}\right) \subset f(B) \subset f(\bar{B}(x, r))$. Therefore $f(z)$ cannot be a point that realizes the diameter of $f(\bar{B}(x, r))$.

Lemma 6.1 together with [10, Theorem 7.2] shows that $f$ satisfies the Lusin condition. 
Corollary 6.2. The coarea formula given in Theorem 2.23 holds also when $f$ is a continuous sense-preserving map in class $N_{\text {loc }}^{1, n}\left(\mathcal{S}, \mathbb{R}^{n}\right)$. This is a consequence of the Lusin condition, Theorem 2.23 and Theorem 2.5 .

Theorem 6.3. A continuous sense-preserving map in $N_{l o c}^{1, n}\left(\mathcal{S}, \mathbb{R}^{n}\right)$ is differentiable almost everywhere.

By Theorem 2.19] it suffices to show that

$$
L_{f}=\left\{x \in \mathcal{S}: \limsup _{y \rightarrow x} \frac{|f(x)-f(y)|}{|x-y|}=\infty\right\}
$$

is a set of measure zero. In order to show this we use the following theorem which is a direct consequence of a more general result [6, Theorem 7.1].

Theorem 6.4. Let $f \in N_{\text {loc }}^{1, n}\left(\mathcal{S}, \mathbb{R}^{n}\right)$ be a continuous map and let $g$ be an upper gradient of $f$. Then the restriction of $f$ to the set $\left\{x:\left|x-x_{0}\right|=r\right\}$ is uniformly Hölder continuous with exponent $\frac{1}{n}$ for almost every $0<r<r_{0}$. In particular, there is a constant $C(\mathcal{S})$ and a radius $r_{0} / 2<r<r_{0}$ such that

$$
|f(x)-f(y)| \leq C(\mathcal{S})|x-y|^{\frac{1}{n}} r_{0}^{\frac{n-1}{n}}\left(f_{B\left(x_{0}, 5 \lambda r_{0}\right)} g(x)^{n} d \mathcal{H}^{n}(x)\right)^{\frac{1}{n}},
$$

whenever $\left|x_{0}-x\right|=\left|x_{0}-y\right|=r$.

Next we give a proof for Theorem 6.3 .

Proof. By Theorem 2.19 it suffices to show that $\mathcal{H}^{n}\left(L_{f}\right)=0$. Fix $x_{0} \in \mathcal{S}, \delta>0$ and $y \in B\left(x_{0}, \delta\right) \backslash\left\{x_{0}\right\}$. Denote $r_{0}=2\left|x_{0}-y\right|$. According to Theorem 6.4 there exists a radius $r, \frac{r_{0}}{2}<r<r_{0}$ such that

$$
|f(x)-f(z)| \leq C|x-z|^{\frac{1}{n}} r_{0}^{\frac{n-1}{n}}\left(f_{B\left(x_{0}, 5 \lambda r_{0}\right)} g(x)^{n} d \mathcal{H}^{n}(x)\right)^{\frac{1}{n}}
$$

for every $x, z$ with $\left|x_{0}-z\right|=\left|x_{0}-x\right|=r$. By Lemma $6.1 f$ is a monotone map i.e., for every $s>0$,

$$
\operatorname{diam} f\left(\bar{B}\left(x_{0}, s\right)\right) \leq \operatorname{diam} f\left(\partial B\left(x_{0}, s\right)\right)
$$

Thus

$$
\left|f\left(x_{0}\right)-f(y)\right| \leq \operatorname{diam} f\left(\partial B\left(x_{0}, r\right)\right) \leq C r_{0}\left(f_{B\left(x_{0}, 5 \lambda r_{0}\right)} g(x)^{n} d \mathcal{H}^{n}(x)\right)^{\frac{1}{n}} .
$$

That is,

$$
\frac{\left|f\left(x_{0}\right)-f(y)\right|}{\left|x_{0}-y\right|} \leq C\left(f_{B\left(x_{0}, 5 \lambda r_{0}\right)} g(x)^{n} d \mathcal{H}^{n}(x)\right)^{\frac{1}{n}},
$$

which is bounded for almost every $x_{0} \in \mathcal{S}$ by the maximal function theorem. 


\section{Openness AND Discreteness}

In this section we finally prove the main result of this paper, Theorem 3.3 . The following lemma is a key tool.

Lemma 7.1. Let $\mathcal{S}$ support a weak $(n-1)$-Poincaré inequality and let $1<n-1<$ $p<n$. Let $E \subset \mathcal{S}$ be a closed set such that there is a sequence $\left\{u_{i}\right\}_{i=1}^{\infty}$ of continuous functions with $n$-weak upper gradients $\left\{g_{i}\right\}_{i=1}^{\infty}$ respectively, satisfying the following conditions.

(1) $u_{i}(x)=1$, for every $i \in \mathbb{N}$ and for every $x \in E$.

(2) $\int_{\Omega} g_{i}(x)^{p} d \mathcal{H}^{n}(x) \rightarrow 0$, as $i \rightarrow \infty$, for every compact set $\Omega \subset \mathcal{S}$.

(3) There exist $y \in \mathcal{S}$ and $R>0$ such that $u_{i}(x) \leq \frac{1}{2}$ for every $x \in \mathcal{S} \cap B(y, R)$ and for every $i \in \mathbb{N}$.

Then $\mathcal{H}_{\infty}^{1}(E)=0$ and thus also $\mathcal{H}^{1}(E)=0$.

The idea of the proof is based on the proof of [9, Theorem 5.9].

Proof. First we show that for every compact set $K \subset \mathcal{S}$ there is $i_{K} \in \mathbb{N}$ such that

$$
\left|u_{i}(x)-\left(u_{i}\right)_{B(x, R)}\right| \geq \frac{1}{4},
$$

whenever $x \in K \cap E$ and $i \geq i_{K}$. For $x \in E \cap K$ set $r_{x}=|x-y|$ and $R_{K}=$ $2(\operatorname{dist}(y, E \cap K)+\operatorname{diam}(E \cap K))$. Note that $R<r_{x}<R_{K}$ for every $x \in E \cap K$. By properties (1) and (3) we have

$$
\begin{aligned}
\frac{1}{2} & \leq\left|u_{i}(x)-\left(u_{i}\right)_{B(y, R)}\right| \\
& \leq\left|u_{i}(x)-\left(u_{i}\right)_{B(x, R)}\right|+\left|\left(u_{i}\right)_{B(x, R)}-\left(u_{i}\right)_{B\left(x, r_{x}\right)}\right| \\
& +\left|\left(u_{i}\right)_{B\left(x, r_{x}\right)}-\left(u_{i}\right)_{B\left(y, r_{x}\right)}\right|+\left|\left(u_{i}\right)_{B\left(y, r_{x}\right)}-\left(u_{i}\right)_{B(y, R)}\right| .
\end{aligned}
$$

On the other hand, by the Poincaré inequality,

$$
\begin{aligned}
\left|\left(u_{i}\right)_{B(x, R)}-\left(u_{i}\right)_{B\left(x, r_{x}\right)}\right| & \leq \frac{C(\mathcal{S})}{R^{n}} r_{x}^{n} f_{B\left(x, r_{x}\right)}\left|u_{i}(z)-\left(u_{i}\right)_{B\left(x, r_{x}\right)}\right| d \mathcal{H}^{n}(z) \\
& \leq \frac{C(\mathcal{S})}{R^{n}} r_{x}^{n+1}\left(f_{B\left(x, \lambda r_{x}\right)} g_{i}(z)^{p} d \mathcal{H}^{n}(z)\right)^{\frac{1}{p}} \\
& \leq \frac{C(\mathcal{S}, p)}{R^{n}} r_{x}^{n+1-\frac{n}{p}}\left(\int_{B\left(x, \lambda r_{x}\right)} g_{i}(z)^{p} d \mathcal{H}^{n}(z)\right)^{\frac{1}{p}} \\
& \leq \frac{C(\mathcal{S}, p)}{R^{n}} R_{K}^{n+1-\frac{n}{p}}\left(\int_{B\left(y, \lambda R_{K}\right)} g_{i}(z)^{p} d \mathcal{H}^{n}(z)\right)^{\frac{1}{p}} .
\end{aligned}
$$

Similarly,

$$
\left|\left(u_{i}\right)_{B\left(y, r_{x}\right)}-\left(u_{i}\right)_{B(y, R)}\right| \leq \frac{C(\mathcal{S}, p)}{R^{n}} R_{K}^{n+1-\frac{n}{p}}\left(\int_{B\left(y, \lambda R_{K}\right)} g_{i}(z)^{p} d \mathcal{H}^{n}(z)\right)^{\frac{1}{p}}
$$

and

$$
\left|\left(u_{i}\right)_{B\left(x, r_{x}\right)}-\left(u_{i}\right)_{B\left(y, r_{x}\right)}\right| \leq \frac{C(\mathcal{S}, p)}{R^{n}} R_{K}^{n+1-\frac{n}{p}}\left(\int_{B\left(y, \lambda R_{K}\right)} g_{i}(z)^{p} d \mathcal{H}^{n}(z)\right)^{\frac{1}{p}} .
$$


Thus by inequality (16) and property (2) we conclude that for each compact $K$ there is $i_{K}$ as stated. Now for a compact set $K$, for every $x \in E \cap K$ and for each $i \geq i_{K}$, we have

$$
\begin{aligned}
\frac{1}{4} \leq\left|u_{i}(x)-\left(u_{i}\right)_{B(x, R)}\right| & \leq \sum_{k=0}^{\infty}\left|\left(u_{i}\right)_{B\left(x, 2^{-k} R\right)}-\left(u_{i}\right)_{B\left(x, 2^{-(k+1)} R\right)}\right| \\
& \leq \sum_{k=0}^{\infty} C R 2^{-k}\left(f_{B\left(x, \lambda 2^{-k} R\right)} g_{i}(z)^{p} d \mathcal{H}^{n}(z)\right)^{\frac{1}{p}} .
\end{aligned}
$$

Let $\epsilon>0$ and denote $\sigma=\frac{-1+p-n}{2}$. Since $p>n-1$ we have $-1<\sigma<p-n$. If for every $k=0,1,2, \ldots$,

$$
\left(f_{B\left(x, \lambda 2^{-k} R\right)} g_{i}(z)^{p} d \mathcal{H}^{n}(z)\right)^{\frac{1}{p}} \leq \frac{1}{R} \epsilon^{\frac{1}{p}} 2^{\frac{\sigma+n}{p} k},
$$

then

$$
\frac{1}{4} \leq \epsilon^{\frac{1}{p}} \sum_{k=1}^{\infty} C 2^{\left(\frac{\sigma+n}{p}-1\right) k} \leq C \epsilon^{\frac{1}{p}}
$$

For sufficiently small $\epsilon$ this is a contradiction. Thus we can choose $\epsilon(\mathcal{S}, p)>0$ to be so small that for every $x \in E \cap K$ there exists an index $k_{x}$ such that

$$
\frac{1}{R} \epsilon^{\frac{1}{p}} 2^{\frac{\sigma+n}{p} k_{x}} \leq\left(f_{B\left(x, \lambda 2^{\left.-k_{x} R\right)}\right.} g_{i}(z)^{p} d \mathcal{H}^{n}(z)\right)^{\frac{1}{p}} .
$$

We apply the basic covering theorem (see [7, Theorem 1.2]) to find a countable collection of pairwise disjoint balls $B_{k}=B\left(x_{k}, 2^{-j_{k}} R\right)$ such that

$$
E \cap K \subset \cup_{k=1}^{\infty} 5 B_{k}
$$

and

Hence

$$
\epsilon \lambda^{n} R^{n-p} 2^{\sigma j_{k}} \leq \int_{B\left(x_{k}, \lambda 2^{-j_{k}} R\right)} g_{i}(z)^{p} d \mathcal{H}^{n}(z)
$$

$$
\begin{aligned}
\frac{1}{5} \mathcal{H}_{\infty}^{1}(E \cap K) & \leq \sum_{k=1}^{\infty} 2^{-j_{k}} R \\
& \leq \sum_{k=1}^{\infty} 2^{-j_{k}} R \frac{1}{\epsilon} \lambda^{-n} R^{p-n} 2^{-\sigma j_{k}} \int_{B\left(x_{k}, \lambda 2^{\left.-j_{k} R\right)}\right.} g_{i}(z)^{p} d \mathcal{H}^{n}(z) \\
& \leq \sum_{k=1}^{\infty} 2^{(-1-\sigma) j_{k}} R^{p+1-n} \lambda^{-n} \frac{1}{\epsilon} \int_{B\left(x_{k}, \lambda 2^{\left.-j_{k} R\right)}\right.} g_{i}(z)^{p} d \mathcal{H}^{n}(z)
\end{aligned}
$$

Here $-1<\sigma$ and thus $2^{(-1-\sigma) j_{k}} \leq 2$ for every $k$. Furthermore, since balls $B_{k}$ are pairwise disjoint the overlap of balls $B\left(x_{k}, \lambda 2^{-j_{k}} R\right)$ is bounded by a constant depending only on $\mathcal{S}$. Thus

$$
\mathcal{H}_{\infty}^{1}(E \cap K) \leq C(\mathcal{S}, p) R^{p+1-n} \int_{B\left(y, \lambda\left(R_{K}+R\right)\right)} g_{i}(z)^{p} d \mathcal{H}^{n}(z) .
$$

Now let $i \rightarrow \infty$ to obtain $\mathcal{H}_{\infty}^{1}(E \cap K)=0$. The claim follows. 
Lemma 7.2. Let $f: \mathcal{S} \rightarrow \mathbb{R}^{n}$ be a sense-preserving map satisfying $\mathcal{H}^{1}\left(f^{-1}(y)\right)=0$ for every $y \in \mathbb{R}^{n}$. Then $f$ is discrete and open.

The idea of the proof is based on the proof of [26, Theorem 2].

Proof. Let $y \in \mathbb{R}^{n}$ and $x \in f^{-1}(y)$. Define $R: \mathcal{S} \rightarrow \mathbb{R}, R(z)=|x-z|$. Then $R$ is 1 -Lipschitz and thus $\mathcal{H}^{1}\left(R\left(f^{-1}(y)\right)\right)=0$. This implies that for $\mathcal{H}^{1}$-almost every $r>0$,

$$
y \notin f(\partial B(x, r)) \subset f(\mathcal{S}(x, r)) .
$$

Assume to the contrary that $f$ is not discrete. Then there is $y_{0} \in \mathbb{R}^{n}$ and $x_{0} \in \mathcal{S}$ such that $f^{-1}\left(y_{0}\right)$ accumulates to $x_{0}$. Fix $r>0$ such that $y_{0} \notin f\left(\partial B\left(x_{0}, r\right)\right)$ and write $B_{0}$ for the $x_{0}$-component of $B\left(x_{0}, r\right)$. Note that $\partial B_{0} \subset \partial B\left(x_{0}, r\right)$. Set $N=$ $\mu\left(y_{0}, f, B_{0}\right)$. Since $f^{-1}\left(y_{0}\right)$ accumulates to $x_{0}$ we find points $x_{1}, x_{2}, \ldots, x_{N+1} \in$ $f^{-1}\left(y_{0}\right) \cap B_{0}$ and radii $r_{i}>0$ such that the balls $B\left(x_{i}, r_{i}\right)$ are pairwise disjoint and

$$
y_{0} \notin \bigcup_{i=1}^{N+1} f\left(\partial B\left(x_{i}, r_{i}\right)\right) \text {. }
$$

Denote the $x_{i}$-components of these balls by $B_{i}$ respectively. Then $y_{0} \notin \bigcup_{i=1}^{N+1} f\left(\partial B_{i}\right)$. Since $B_{0} \cap f^{-1}\left(y_{0}\right) \backslash \bigcup_{i=1}^{N+1} B_{i}=\bar{B}_{0} \cap f^{-1}\left(y_{0}\right) \backslash \bigcup_{i=1}^{N+1} B_{i}$ is compact and the components of $B_{0} \backslash \bigcup_{i=1}^{N+1} \bar{B}_{i}$ cover it, we find a finite subcover $\left\{U_{i}\right\}_{i=1}^{p}$. We use part (1) of Lemma 2.33 and the fact that $f$ is sense-preserving to conclude

$$
N=\mu\left(y_{0}, f, B_{0}\right)=\sum_{i=1}^{N+1} \mu\left(y_{0}, f, B_{i}\right)+\sum_{i=1}^{p} \mu\left(y_{0}, f, U_{i}\right) \geq \sum_{i=1}^{N+1} \mu\left(y_{0}, f, B_{i}\right) \geq N+1 .
$$

This is a contradiction and thus $f$ is discrete. Next we show that $f$ is open. Let $U \subset$ $\mathcal{S}$ be open and $x \in U$. We find ball $B(x, r) \subset U$ such that $f(x) \notin f(\partial B(x, r))$. Let $B_{0}$ denote the $x$-component of $B(x, r)$. Now $\mu\left(y, f, B_{0}\right)$ is a positive and constant on the $f(x)$-component of $f\left(\partial B_{0}\right)^{C}$. Thus the $f(x)$-component of $f\left(\partial B_{0}\right)^{C}$ is included in $f\left(B_{0}\right) \subset f(U)$. On the other hand, the $f(x)$-component is open. Thus $f(x)$ lies in the interior of $f(U)$ and therefore $f$ is open.

From now on, unless otherwise stated, a mapping denoted by $f$ is assumed to be a mapping of finite distortion as in Theorem 3.3. According to Lemma 7.2 , for discreteness and openness it is sufficient to show that for every $y \in \mathbb{R}^{n}$ the Hausdorff 1-measure of $f^{-1}(y)$ is zero and thus it suffices to find a sequence of functions as in Lemma 7.1. By translation we may consider only the case $y=0$.

Lemma 7.3. Fix $s>0$ and let $u: \mathcal{S} \rightarrow[0, \infty]$,

$$
u(x)= \begin{cases}\left(\log ^{+} \log ^{+} \frac{1}{|f(x)|}\right)^{s} & \text { if } x \in \mathcal{S} \backslash f^{-1}(0), \\ \infty & \text { if } x \in f^{-1}(0)\end{cases}
$$

Then $u$ is differentiable almost everywhere in the set $\mathcal{S} \backslash f^{-1}(0)$ and $\|D u\|$ is an $n$-weak upper gradient of $u$.

Proof. For each $i \in \mathbb{N}$ write $A_{i}=f^{-1}\left(\bar{B}\left(0, \frac{1}{i}\right)\right)$. Because for each $i$ the function $h_{i}: \bar{B}\left(0, \frac{1}{i}\right)^{c} \rightarrow\left[0, \infty\left[, h_{i}(x)=\left(\log ^{+} \log ^{+} \frac{1}{|x|}\right)^{s}\right.\right.$ is Lipschitz and $f \in N_{l o c}^{1, n}\left(\mathcal{S}, \mathbb{R}^{n}\right)$ we conclude that $u_{i}=h_{i} \circ f \in N_{l o c}^{1, n}\left(\mathcal{S} \backslash A_{i}\right)$. Furthermore, $u_{i}$ is continuous and differentiable almost everywhere in the open set $\mathcal{S} \backslash A_{i}$, and $\left\|D u_{i}(\cdot)\right\|=\|D u(\cdot)\|_{\mid \mathcal{S} \backslash A_{i}}$. 
Thus for $n$-almost every rectifiable path $\gamma$ in $\mathcal{S} \backslash A_{i}$ the function $u_{i} \circ \gamma$ is absolutely continuous. Let $\gamma:\left[0, l_{\gamma}\right] \rightarrow \mathcal{S} \backslash A_{i}$ be such a path, parametrized by arc length. Then

$$
\left|\left(u_{i} \circ \gamma\right)(0)-\left(u_{i} \circ \gamma\right)\left(l_{\gamma}\right)\right| \leq \int_{0}^{l_{\gamma}}\left|\left(u_{i} \circ \gamma\right)^{\prime}(t)\right| d t .
$$

If $u_{i} \circ \gamma$ is differentiable at $t$ and $\gamma(t) \in \mathcal{S} \backslash P_{u}$, we have

$$
\left|\left(u_{i} \circ \gamma\right)^{\prime}(t)\right|=\lim _{s \rightarrow t} \frac{\left|\left(u_{i} \circ \gamma\right)(s)-\left(u_{i} \circ \gamma\right)(t)\right|}{|\gamma(s)-\gamma(t)|} \leq \operatorname{Lip} u(\gamma(t))=\|D u(\gamma(t))\| .
$$

Here the last equality follows from Lemma 2.28. We conclude that $\gamma$ and $\left\|D u_{i}(\cdot)\right\|$ satisfy the upper gradient inequality (10). Furthermore since $u$ is continuous it follows from Lemma 2.28 that $\|D u(\cdot)\|$ is a Borel function. Thus also $\left\|D u_{i}(\cdot)\right\|=$ $\|D u(\cdot)\|_{\mid \mathcal{S} \backslash A_{i}}$ is a Borel function. Finally we conclude that $\left\|D u_{i}(\cdot)\right\|$ is an $n$-weak upper gradient of $u_{i}$. Now, for each $i \in \mathbb{N}$ there is $\Gamma_{i}$ such that $\operatorname{Mod}_{n} \Gamma_{i}=0$ and if $\gamma \notin \Gamma_{i}$ is a rectifiable path in $\mathcal{S} \backslash A_{i}$ then $\gamma$ and $\|D u(\cdot)\|$ satisfy the upper gradient inequality (11). Let $\Gamma_{0}$ be the collection of all rectifiable paths in $\mathcal{S}$ that have a subpath in some $\Gamma_{i}$. Then $\operatorname{Mod}_{n} \Gamma_{0}=0$. Let $\gamma:\left[0, l_{\gamma}\right] \rightarrow \mathcal{S}$ be a rectifiable path, $\gamma \notin \Gamma_{0}$, parametrized by arc length. We will show that $\gamma$ and $\|D u(\cdot)\|$ satisfy the upper gradient inequality (11). We may assume that $\gamma^{-1}\left(f^{-1}(0)\right)=\left\{l_{\gamma}\right\}$. Let $\left\{a_{n}\right\}_{n=1}^{\infty} \subset\left[0, l_{\gamma}\left[\right.\right.$ such that $a_{n} \rightarrow l_{\gamma}$, as $n \rightarrow \infty$. Since $\gamma$ does not have a subpath in $\Gamma_{0}$, we get

$$
\begin{aligned}
& |u(\gamma(0))-u(\gamma(a))|=\lim _{n \rightarrow \infty}\left|u(\gamma(0))-u\left(\gamma\left(a_{n}\right)\right)\right| \\
& \leq \lim _{n \rightarrow \infty} \int_{\gamma_{\mid\left[0, a_{n}\right]}}\|D u(\cdot)\| d t \leq \int_{\gamma}\|\operatorname{apD} D(\cdot)\| d t .
\end{aligned}
$$

Therefore $\|D u(\cdot)\|$ is an $n$-weak upper gradient of $u$.

Remark 7.4. If we change the value of $\|D u\|$ to be 0 in the set $u^{-1}(\infty)$ the resulting function is still an $n$-weak upper gradient of $u$. That is, the function $g: \mathcal{S} \rightarrow[0, \infty]$,

$$
g(x)= \begin{cases}\|D u(x)\| & \text { if } x \in \mathcal{S} \backslash f^{-1}(0), \\ 0 & \text { if } x \in f^{-1}(0),\end{cases}
$$

is an $n$-weak upper gradient of $u$. Next we explain why good local integrability of the function $g$ implies that $\mathcal{H}^{1}\left(f^{-1}(0)\right)=0$. For every $i \in \mathbb{N}$ define

$$
u_{i}(x)=\frac{1}{i} \min \{u(x), i\} .
$$

Then $u_{i}(x)=1$ for every $x \in f^{-1}(0)$. Because $f$ is continuous and not a constant function there is a ball $B(y, R) \subset \mathcal{S}$ such that when $i$ is sufficiently large we have $u_{i}(x) \leq \frac{1}{2}$, for every $x \in B(y, R)$. On the other hand, for every $i$ the function $\frac{1}{i} g$ is an $n$-weak upper gradient of $u_{i}$. Now if $g^{p}$ is locally integrable for some $n-1<p<n$ we can apply Lemma 7.1 to obtain $\mathcal{H}^{1}\left(f^{-1}(0)\right)=0$. To show that $n$-weak upper gradient $g$ is locally $p$-integrable we establish the following estimates. We write $h(x)=\left(\log ^{+} \log ^{+} \frac{1}{|x|}\right)^{s}$. Since $u$ is differentiable almost everywhere in 
the set $\mathcal{S} \backslash f^{-1}(0)$ we have

$$
\begin{aligned}
g(x)=\|D u(x)\| & =\operatorname{Lip} u(x)=\limsup _{y \rightarrow x} \frac{|(h \circ f)(y)-(h \circ f)(x)|}{|y-x|} \\
& \leq \limsup _{y \rightarrow x} \frac{|(h \circ f)(y)-(h \circ f)(x)|}{|f(y)-f(x)|} \limsup _{y \rightarrow x} \frac{|f(y)-f(x)|}{|y-x|} \\
& \leq C(n) s \frac{\left(\log \log \frac{1}{|f(x)|}\right)^{s-1}}{|f(x)| \log \left(\frac{1}{|f(x)|}\right)}\|D f(x)\|
\end{aligned}
$$

for almost every $x \in \mathcal{S} \backslash f^{-1}(0)$. Let $\Omega \subset \mathcal{S}$ be a compact set. The distortion inequality and Hölder's inequality together imply that for any $n-1<p<n$,

$$
\begin{aligned}
& \int_{\Omega \backslash f^{-1}(0)}\left(s \frac{\left(\log \log \frac{1}{|f|}\right)^{s-1}}{|f| \log \left(\frac{1}{|f|}\right)}\|D f\|\right)^{p} d \mathcal{H}^{n}(x) \\
& \leq\left(\int_{\Omega \backslash f^{-1}(0)} K^{\frac{p}{n-p}} d \mathcal{H}^{n}(x)\right)^{\frac{n-p}{n}} \\
& \quad \times\left(\int_{\Omega \backslash f^{-1}(0)} \frac{s^{n}}{|f|^{n} \log ^{n} \frac{1}{|f|}}\left(\log \log \frac{1}{|f|}\right)^{(s-1) n} J_{f} d \mathcal{H}^{n}(x)\right)^{\frac{p}{n}} .
\end{aligned}
$$

Here

$$
\begin{aligned}
& \int_{\Omega \backslash f^{-1}(0)} \frac{s^{n}}{|f|^{n} \log ^{n} \frac{1}{|f|}}\left(\log \log \frac{1}{|f|}\right)^{(s-1) n} J_{f} d \mathcal{H}^{n}(x) \\
& \leq \int_{f(\Omega)} \frac{s^{n}}{|y|^{n} \log ^{n} \frac{1}{|y|}}\left(\log \log \frac{1}{|y|}\right)^{(s-1) n} \operatorname{card} f^{-1}(y) d \mathcal{H}^{n}(y) .
\end{aligned}
$$

We assume that $K \in L_{l o c}^{q}(\mathcal{S})$, for some $q>n-1$. By this assumption we find $p(q)>n-1$ such that the first integral on the right-hand side of (17) is finite. In order to prove that $g$ is locally $p$-integrable we need to show that the last integral on the right-hand side is finite as well. In 21, Onninen and Zhong considered the function $g$ with parameter $s=1$. They showed that $g$ is $p$-integrable and integration by parts was a crucial tool. Instead of integration by parts, we use the path lifting property of branched covers to give a bound for $\operatorname{card} f^{-1}(y)$ in (18). This is done in Lemma 7.9. However, in order to use path lifting we need some preparations. First we show that any point $x$ satifying $\mathcal{H}^{1}\left(f^{-1}(f(x))\right)=0$ has a neighborhood $Z$ such that $f$ restricted to $Z$ is a branched cover. In order to do this we again employ Lemma 7.1 and the function $g$ introduced above, but in this setting we can use the theory of the topological degree and coarea formula to show that $\operatorname{card} f^{-1}(y)$ is essentially bounded. This is made precise in the following lemma.

Lemma 7.5. Let $y_{0} \in \mathbb{R}^{n}$ such that $\mathcal{H}^{1}\left(f^{-1}\left(y_{0}\right)\right)=0$, and let $x_{0} \in f^{-1}\left(y_{0}\right)$. Then $f$ is discrete and open on some connected neighborhood of $x_{0}$.

Proof. According to Lemma 7.2, it suffices to find an open and connected neighborhood $Z$ of $x_{0}$ such that $\mathcal{H}^{1}\left(f^{-1}(f(z)) \cap Z\right)=0$, for every $z \in Z$. As in the proof of Lemma 7.2 we find $r>0$ such that $y_{0} \notin f\left(\partial B\left(x_{0}, r\right)\right)$. Write $Z$ for the $x_{0}$-component of $B\left(x_{0}, r\right)$. Then $Z$ is an open and connected neighborhood of $x_{0}$ and $y_{0} \notin f(\partial Z)$. Denote the $y_{0}$-component of $\mathbb{R}^{n} \backslash f(\partial Z)$ by $Y$. Let $z \in Z$. In order to show that $\mathcal{H}^{1}\left(f^{-1}(f(z)) \cap Z\right)=0$ we may use a translation and assume that 
$z=0=f(z)$. According to Remark 7.4 it suffices to show that the function $g$ is $p$-integrable on $Z$. In the definition of $g$ we may choose $s=1$. Finally, by inequality (17) we are left to show that

$$
\int_{Z \backslash f^{-1}(0)} \frac{J_{f}(x)}{|f(x)|^{n} \log ^{n} \frac{1}{|f(x)|}} d \mathcal{H}^{n}(x)<\infty .
$$

Denote $A=\left\{x \in Z: \operatorname{card}\left\{f^{-1}(f(x)) \cap Z\right\}=\infty\right\}$. Because $J_{f}$ is locally integrable the coarea formula gives

$$
\int_{f(A)} \operatorname{card}\left\{f^{-1}(y) \cap A\right\} d \mathcal{H}^{n}(y)=\int_{A} J_{f}(x) d \mathcal{H}^{n}(x)<\infty .
$$

Thus $J_{f}(x)=0$, for almost every $x \in A$. We will show that $\operatorname{card} f^{-1}(y) \cap Z$ is uniformly bounded on $Y \backslash f(A)$. Fix $y \in Y \backslash f(A)$ and denote card $f^{-1}(y) \cap Z$ by $N_{y}$. Since $N_{y}$ is finite there is collection $\left\{U_{i}\right\}_{i=1}^{N_{y}}$ of open connected disjoint subsets of $Z$ such that each $U_{i}$ contains exactly one point of $f^{-1}(y) \cap Z$ and for each $i$ we have $y \notin f\left(\partial U_{i}\right)$. Since $f$ is sense-preserving and the map $\omega \mapsto \mu(\omega, f, Z)$ is constant on $Y$, we have

$$
N_{y} \leq \sum_{i=1}^{N_{y}} \mu\left(y, f, U_{i}\right)=\mu(y, f, Z)=\mu\left(y_{0}, f, Z\right) .
$$

In particular, for every $y \in f(Z \backslash A)$,

$$
\operatorname{card}\left\{f^{-1}(y) \cap Z\right\} \leq \mu\left(y_{0}, f, Z\right) .
$$

Thus

$$
\begin{aligned}
& \int_{Z \backslash f^{-1}(0)} \frac{J_{f}(x)}{|f(x)|^{n} \log ^{n} \frac{1}{|f(x)|}} d \mathcal{H}^{n}(x)=\int_{Z \backslash f^{-1}(0) \backslash A} \frac{J_{f}(x)}{|f(x)|^{n} \log ^{n} \frac{1}{|f(x)|}} d \mathcal{H}^{n}(x) \\
& \leq \int_{f(Z \backslash A)} \frac{\operatorname{card}\left\{f^{-1}(y) \cap(Z \backslash A)\right\}}{|y|^{n} \log ^{n} \frac{1}{|y|}} d \mathcal{H}^{n}(y) \\
& \leq \mu\left(y_{0}, f, B\left(x_{0}, r\right)\right) \int_{f(Z \backslash A)} \frac{1}{|y|^{n} \log ^{n} \frac{1}{|y|}} d \mathcal{H}^{n}(y),
\end{aligned}
$$

but this is finite.

The following lemma is a modification of [22, Lemma 2.5]. It tells that there is a large set of good points in the sense of Lemma 7.5. In what follows, we will use the spherical coordinate system in $\mathbb{R}^{n}$. That is, $z=(\varphi, t)$, where $\varphi=\frac{z}{|z|}$ and $t=|z|$.

Lemma 7.6. Let $f: \mathcal{S} \rightarrow \mathbb{R}^{n}$ be a mapping of finite distortion and let

$$
E=\left\{\varphi \in \mathcal{S}^{n-1}(0,1): \mathcal{H}^{1}\left(f^{-1}(\varphi, t)\right)>0, \text { for some } t>0\right\} .
$$

Then $\mathcal{H}^{n-1}(E)=0$.

Proof. Fix $R>0$ and write

$$
E_{R}=\left\{\varphi \in \mathcal{S}^{n-1}(0,1): \mathcal{H}^{1}\left(f^{-1}(\varphi, t) \cap B(0, R)\right)>0, \text { for some } t>0\right\} .
$$

Denote by $Z$ the set

$$
Z=\left\{x \in \mathcal{S}: \mathcal{H}^{1}\left(f^{-1}(f(x)) \cap B(0, R)\right)>0\right\}
$$


and let

$$
E_{i}=\left\{\varphi \in E_{R}: \mathcal{H}^{1}\left(f^{-1}(\varphi, t) \cap B(0, R) \cap Z\right)>\frac{1}{i}, \text { for some } t>0\right\} .
$$

Then $E_{R}=\bigcup_{i=1}^{\infty} E_{i}$ and by the coarea formula

$$
\begin{aligned}
\frac{1}{i} \mathcal{H}^{n-1}\left(E_{i}\right) & \leq \int_{E_{i}} \mathcal{H}^{1}\left(f^{-1}(\varphi \times(0, \infty)) \cap Z \cap B(0, R)\right) d \mathcal{H}^{n-1}(\varphi) \\
& \leq \int_{Z \cap B(0, R)} J_{\pi \circ f}(x) d \mathcal{H}^{n}(x) .
\end{aligned}
$$

Here $\pi$ denotes the projection $\pi: \mathbb{R}^{n} \backslash\{0\} \rightarrow \mathcal{S}^{n-1}(0,1), \pi(x)=\frac{x}{|x|}$. On the other hand, since $J_{f}$ is locally integrable the coarea formula gives

$$
\int_{f(Z \cap B(0, R))} \operatorname{card}\left\{f^{-1}(y) \cap Z \cap B(0, R)\right\} d \mathcal{H}^{n}(y)=\int_{Z \cap B(0, R)} J_{f}(x) d \mathcal{H}^{n}(x)<\infty .
$$

Since $\mathcal{H}^{1}\left(f^{-1}(y) \cap Z \cap B(0, R)\right)>0$ for every $y \in f(Z \cap B(0, R))$ we conclude that $\mathcal{H}^{n}(f(Z \cap B(0, R)))=0$ and thus $J_{f}(x)=0$ for almost every $x \in Z \cap B(0, R)$. By the distortion inequality (12) this implies that $\|D f(x)\|=0$ and thus $J_{\pi \circ f}(x)=0$ for almost every $x \in Z \cap B(0, R)$. Thus $\mathcal{H}^{n}\left(E_{i}\right)=0$ for every $i$ and so also $\mathcal{H}^{n}\left(E_{R}\right)=0$. The claim follows.

Corollary 7.7. According to Lemmas 7.5] and 7.6, for $\mathcal{H}^{n-1}$-almost every $\varphi \in$ $\mathcal{S}^{n-1}(0,1)$ the preimage of a radial path $\{(\varphi, t), t>0\}$ has an open neighborhood $U_{\varphi}$ such that $f$ restricted to $U_{\varphi}$ is open and discrete. We denote the set of such points $\varphi \in \mathcal{S}^{n-1}(0,1)$ by $A$.

Lemma 7.8. Let $f: \mathcal{S} \rightarrow \mathbb{R}^{n}$ be a continuous map that is differentiable at $x_{0} \in \mathcal{S}$ with $J_{f}\left(x_{0}\right)>0$. Assume that $f$ defines a branched cover in some neighborhood of $x_{0}$. Then $i\left(x_{0}, f\right)=1$.

Proof. Since $f$ defines a branched cover on some neighborhood $U$ of $x_{0}$, the local index $i\left(x_{0}, f\right)$ is well defined and we find the domain $D \subset U$ such that $x_{0} \in D \subset U$ with

$$
f^{-1}\left(f\left(x_{0}\right)\right) \cap \bar{D}=\left\{x_{0}\right\} .
$$

Because $f$ is differentiable at $x_{0}$ we find the domain $U^{\prime} \subset D$ such that

$$
\mu\left(f\left(x_{0}\right), f, U^{\prime}\right)=\mu\left(f\left(x_{0}\right), D f\left(x_{0}\right) \pi_{x_{0}}, U^{\prime}\right) .
$$

Furthermore, since $\mathcal{S}$ is metrically oriented and $J_{f}\left(x_{0}\right)>0$ we may assume that $U^{\prime}$ is chosen such that

$$
\mu\left(x_{0}, \pi_{x_{0}}, U^{\prime}\right)=1=\mu\left(x_{0}, D f\left(x_{0}\right), \pi_{x_{0}} U^{\prime}\right) .
$$

But then $\mu\left(x_{0}, D f\left(x_{0}\right) \pi_{x_{0}}, U^{\prime}\right)=1$ and hence $\mu\left(f\left(x_{0}\right), f, U^{\prime}\right)=1$. According to the definition of the local index this proves the claim.

As pointed out in inequality (18), an upper bound for the multiplicity function is needed in order to show that the function $g$ defined in Remark 7.4 is $p$-integrable. The following lemma gives a proper bound. The idea of the proof is similar to one in the proof of [23, Lemma IV 1.3]. 
Lemma 7.9. Let $f: \mathcal{S} \rightarrow \mathbb{R}^{n}$ be a mapping of finite distortion with $K \in L_{\text {loc }}^{n-1}(\mathcal{S})$. For every $x_{0} \in \mathcal{S}$ and for sufficiently small $r, R>0$ we have

$$
\begin{aligned}
& f_{S^{n-1}\left(f\left(x_{0}\right), r\right)} \operatorname{card}\left\{f^{-1}(y) \cap B\left(x_{0}, R\right)\right\} d \mathcal{H}^{n-1}(y) \\
& \leq C(\mathcal{S}) f_{B\left(x_{0}, 2 R\right) \backslash B\left(x_{0}, R\right)} K(x)^{n-1} d \mathcal{H}^{n}(x) \log ^{n-1} \frac{1}{r} .
\end{aligned}
$$

Proof. We may assume that $x_{0}=f\left(x_{0}\right)=0$. Let $A \subset \mathcal{S}^{n-1}(0,1)$ as in Corollary 7.7 and let $y \in A$. According to Corollary 7.7 the preimage of the radial line associated to $y$ has a neighborhood $U_{y}$ such that $f$ defines a branched cover on it. Thus $f$ defines a branched cover on every connected component of $U_{y}$. On the other hand, every such component is a generalized $n$-manifold, and thus the path lifting property of branched covers applies for radial paths associated to points in $A$. Fix $r, R>0$. For $y \in A$ define

$$
m(y)=\max \left\{0, \sum_{f^{-1}(r y) \cap B(0, R)} i(x, f)-\sum_{f^{-1}(y) \cap B(0,2 R)} i(x, f)\right\},
$$

and for every $k \in\{0,1,2 \ldots\}$ set

$$
A_{k}=\{y \in A: m(y)=k\} .
$$

Then $A=\bigcup_{k=0}^{\infty} A_{k}$ and the sets $A_{k}$ are pairwise disjoint. Fix $k \geq 1$. For each $y \in A_{k}$ define $\beta_{y}:[r, 1) \rightarrow \mathbb{R}^{n}, \beta_{y}(t)=t y$. Then every $\beta_{y}$ has at least $k$ lifts connecting $\bar{B}(0, R)$ to $B(0,2 R)^{C}$. Let $\left\{\gamma_{i}^{y}\right\}_{i=1}^{k}$ be a set of such lifts. Set

$$
E_{k}=\bigcup_{y \in A_{k}} \bigcup_{i=1}^{k}\left|\gamma_{i}^{y}\right| \text {. }
$$

Note that $\mathcal{H}^{1}\left(\left|\gamma_{i}^{y}\right|\right) \geq R$ for every $\gamma_{i}^{y}$. Because for every $y \in A \backslash A_{0}$ at most $i(x, f)$ of the lifts of $\beta_{y}$ pass throught $x$ (see Section 2.5), we have

$$
\begin{aligned}
R k \mathcal{H}^{n-1}\left(A_{k}\right) & \leq \int_{A_{k}} \sum_{i=1}^{k} \mathcal{H}^{1}\left(\left|\gamma_{i}^{y}\right|\right) d \mathcal{H}^{n-1}(y) \\
& =\int_{A_{k}} \int_{\bigcup_{i=1}^{k}\left|\gamma_{i}^{y}\right|} i(x, f) d \mathcal{H}^{1}(x) d \mathcal{H}^{n-1}(y) \\
& \leq \int_{A_{k}} \int_{\left(\frac{f}{|f|}\right)^{-1}(y)} i(x, f) \chi_{E_{m}}(x) d \mathcal{H}^{1}(x) d \mathcal{H}^{n-1}(y) .
\end{aligned}
$$

By the coarea formula and the distortion inequality we have

$$
\begin{aligned}
& \int_{A_{k}} \int_{\left(\frac{f}{|f|}\right)^{-1}(y)} i(x, f) \chi_{E_{m}}(x) d \mathcal{H}^{1}(x) d \mathcal{H}^{n-1}(y) \\
& =\int_{E_{k}} J_{\frac{f}{|f|}}(x) i(x, f) d \mathcal{H}^{n}(x) \leq C(n) \int_{E_{k}} \frac{\|D f(x)\|^{n-1}}{|f(x)|^{n-1}} i(x, f) d \mathcal{H}^{n}(x) \\
& \leq\left(\int_{E_{k}} K(x)^{n-1} d \mathcal{H}^{n}(x)\right)^{\frac{1}{n}}\left(\int_{E_{k}} \frac{J_{f}(x)}{|f(x)|^{n}} i(x, f)^{\frac{n}{n-1}} d \mathcal{H}^{n}(x)\right)^{\frac{n-1}{n}} .
\end{aligned}
$$


Because $f$ is differentiable almost everywhere and because $J_{f}(x) \geq 0$ for almost every $x \in \mathcal{S}$, Lemma 7.8 and change of variables give

$$
\begin{aligned}
& \int_{E_{k}} \frac{J_{f}(x)}{|f(x)|^{n}} i(x, f)^{\frac{n}{n-1}} d \mathcal{H}^{n}(x)=\int_{E_{k}} \frac{J_{f}(x)}{|f(x)|^{n}} d \mathcal{H}^{n}(x) \\
& =\int_{f\left(E_{k}\right)} \frac{\operatorname{card}\left\{f^{-1}(y) \cap E_{k}\right\}}{|y|^{n}} d \mathcal{H}^{n}(x) \\
& =\int_{A_{k} \times[r, 1)} \frac{k}{|y|^{n}} d \mathcal{H}^{n}(y)=k \mathcal{H}^{n-1}\left(A_{k}\right) \log \frac{1}{r} .
\end{aligned}
$$

From (19), (20) and (21) we conclude

$$
\mathcal{H}^{n-1}\left(A_{k}\right) k \leq \frac{C(n)}{R^{n}} \int_{E_{k}} K(x)^{n-1} d \mathcal{H}^{n}(x) \log ^{n-1} \frac{1}{r}
$$

Recall that $\mathcal{H}^{n-1}\left(\mathcal{S}^{n-1}(0,1) \backslash A\right)=0$ and $m(y)=0$, for every $y \in A_{0}$. Thus we sum over $k$ to get

$$
\int_{\mathcal{S}^{n-1}(0,1)} m(y) d \mathcal{H}^{n-1}(y) \leq C(\mathcal{S}) f_{B(0,2 R) \backslash B(0, R))} K(x)^{n-1} d \mathcal{H}^{n}(x) \log ^{n-1} \frac{1}{r} .
$$

Because $f$ is continuous we may choose $R>0$ so small that $f(B(0,2 R)) \subset B\left(0, \frac{1}{2}\right)$. Then $f^{-1}(y) \cap B(0,2 R)=\emptyset$ for every $y \in \mathcal{S}^{n-1}(0,1)$. Since $f$ is sense-preserving, $i(x, f) \geq 1$ whenever defined. Hence

$$
\begin{aligned}
& \int_{\mathcal{S}^{n-1}(0,1)} \operatorname{card}\left\{f^{-1}(r y) \cap B(0, R)\right\} d \mathcal{H}^{n-1}(y) \\
& \leq \int_{\mathcal{S}^{n-1}(0,1)} \max \left\{0, \sum_{f^{-1}(r y) \cap B(0, R)} i(x, f)\right\} d \mathcal{H}^{n-1}(y)=\int_{\mathcal{S}^{n-1}(0,1)} m(y) d \mathcal{H}^{n-1}(y) \\
& \leq C(\mathcal{S}) f_{B(0,2 R) \backslash B(0, R)} K(x)^{n-1} d \mathcal{H}^{n}(x) \log ^{n-1} \frac{1}{r} .
\end{aligned}
$$

The claim follows.

Now we are ready to show that the $n$-weak upper gradient given in Remark 7.4 is locally $p$-integrable. By inequality (17) it suffices to show that

$$
\int_{\Omega \backslash f^{-1}(0)} \frac{s^{n}}{|f(x)|^{n} \log ^{n} \frac{1}{|f(x)|}}\left(\log \log \frac{1}{|f(x)|}\right)^{(s-1) n} J_{f}(x) d \mathcal{H}^{n}(x)<\infty,
$$

for some $s>0$ and for a compact set $\Omega \subset \mathcal{S}$. We may assume that $f(0)=0$ and $\Omega \subset B(0, R)$, where $R$ is as in Lemma 7.9], and $f(B(0, R)) \subset B\left(0, \frac{1}{2 e}\right)$. We use the 
change of variables and Lemma 7.9 as follows:

$$
\begin{aligned}
& \int_{\Omega \backslash f^{-1}(0)} \frac{s^{n}}{|f(x)|^{n} \log ^{n} \frac{1}{|f(x)|}}\left(\log \log \frac{1}{|f(x)|}\right)^{(s-1) n} J_{f}(x) d \mathcal{H}^{n}(x) \\
& =\int_{B\left(0, \frac{1}{2 e}\right)} \frac{s^{n}}{|y|^{n} \log ^{n} \frac{1}{|y|}}\left(\log \log \frac{1}{|y|}\right)^{(s-1) n} \operatorname{card}\left\{f^{-1}(y) \cap B(0, R)\right\} d \mathcal{H}^{n}(y) \\
& \leq \int_{0}^{\frac{1}{2 e}} \frac{s^{n}}{r \log ^{n} \frac{1}{r}}\left(\log \log \frac{1}{r}\right)^{(s-1) n} f_{\mathcal{S}(0, r)} \operatorname{card}\left\{f^{-1}(y) \cap B(0, R)\right\} d \mathcal{S}^{n-1}(y) d r \\
& \leq \int_{0}^{\frac{1}{2 e}} \frac{s^{n}}{r \log ^{n} \frac{1}{r}}\left(\log \log \frac{1}{r}\right)^{(s-1) n} \log ^{n-1} \frac{1}{r} d r<\infty,
\end{aligned}
$$

whenever $(s-1) n+1<0$. So, for example, we may choose $s=\frac{n-1}{2 n}$. Then $g$ is locally $p$-integrable and thus Theorem 3.3 follows from Remark 7.4 and Lemma 7.2

\section{ACKNOWLEDGEMENTS}

The author wishes to thank Professor Kai Rajala for many ideas and helpful discussions. Part of this research was done when the author was visiting at the University of Michigan. He wishes to thank the department for its hospitality. This paper is part of the author's Ph.D. thesis.

\section{REFERENCES}

[1] Daniel Aalto and Juha Kinnunen, The discrete maximal operator in metric spaces, J. Anal. Math. 111 (2010), 369-390, DOI 10.1007/s11854-010-0022-3. MR2747071 (2012a:42027)

[2] J. M. Ball, Global invertibility of Sobolev functions and the interpenetration of matter, Proc. Roy. Soc. Edinburgh Sect. A 88 (1981), no. 3-4, 315-328, DOI 10.1017/S030821050002014X. MR616782(83f:73017)

[3] Zoltán M. Balogh, Kevin Rogovin, and Thomas Zürcher, The Stepanov differentiability theorem in metric measure spaces, J. Geom. Anal. 14 (2004), no. 3, 405-422, DOI 10.1007/BF02922098. MR2077159 (2005d:28008)

[4] J. Cheeger, Differentiability of Lipschitz functions on metric measure spaces, Geom. Funct. Anal. 9 (1999), no. 3, 428-517, DOI 10.1007/s000390050094. MR1708448 (2000g:53043)

[5] Herbert Federer, Geometric measure theory, Die Grundlehren der mathematischen Wissenschaften, Band 153, Springer-Verlag New York Inc., New York, 1969. MR0257325 (41 \#1976)

[6] Piotr Hajłasz and Pekka Koskela, Sobolev met Poincaré, Mem. Amer. Math. Soc. 145 (2000), no. $688, \mathrm{x}+101$, DOI 10.1090/memo/0688. MR.1683160 (2000j:46063)

[7] Juha Heinonen, Lectures on analysis on metric spaces, Universitext, Springer-Verlag, New York, 2001. MR 1800917 (2002c:30028)

[8] Juha Heinonen and Stephen Keith, Flat forms, bi-Lipschitz parameterizations, and smoothability of manifolds, Publ. Math. Inst. Hautes Études Sci. 113 (2011), 1-37, DOI 10.1007/s10240-011-0032-4. MR2805596(2012h:30194)

[9] Juha Heinonen and Pekka Koskela, Quasiconformal maps in metric spaces with controlled geometry, Acta Math. 181 (1998), no. 1, 1-61, DOI 10.1007/BF02392747. MR1654771 (99j:30025)

[10] Juha Heinonen, Pekka Koskela, Nageswari Shanmugalingam, and Jeremy T. Tyson, Sobolev classes of Banach space-valued functions and quasiconformal mappings, J. Anal. Math. 85 (2001), 87-139, DOI 10.1007/BF02788076. MR.1869604(2002k:46090)

[11] J. Heinonen and P. Koskela, N. Shanmugalingam, J. Tyson, Sobolev Spaces on metric measure spaces: an approach based on upper gradients, to appear.

[12] Juha Heinonen and Seppo Rickman, Geometric branched covers between generalized manifolds, Duke Math. J. 113 (2002), no. 3, 465-529, DOI 10.1215/S0012-7094-02-11333-7. MR.1909607 (2003h:57003) 
[13] Juha Heinonen and Dennis Sullivan, On the locally branched Euclidean metric gauge, Duke Math. J. 114 (2002), no. 1, 15-41, DOI 10.1215/S0012-7094-02-11412-4. MR.1915034 (2004b:30044)

[14] Stanislav Hencl and Kai Rajala, Optimal assumptions for discreteness, Arch. Ration. Mech. Anal. 207 (2013), no. 3, 775-783, DOI 10.1007/s00205-012-0574-8. MR3017286

[15] Tadeusz Iwaniec and Vladimír Šverák, On mappings with integrable dilatation, Proc. Amer. Math. Soc. 118 (1993), no. 1, 181-188, DOI 10.2307/2160025. MR1160301 (93k:30023)

[16] Pekka Koskela and Paul MacManus, Quasiconformal mappings and Sobolev spaces, Studia Math. 131 (1998), no. 1, 1-17. MR.1628655(99e:46042)

[17] J. Maly, A simple proof of the Stepanov theorem on differentiability almost everywhere, Exposition. Math. 17 (1999), no. 1, 59-61. MR1687460(2000a:26012)

[18] Juan J. Manfredi and Enrique Villamor, Mappings with integrable dilatation in higher dimensions, Bull. Amer. Math. Soc. (N.S.) 32 (1995), no. 2, 235-240, DOI 10.1090/S0273-09791995-00583-5. MR:1313107 (95m:30033)

[19] Pertti Mattila, Geometry of sets and measures in Euclidean spaces: Fractals and rectifiability, Cambridge Studies in Advanced Mathematics, vol. 44, Cambridge University Press, Cambridge, 1995. MR1333890 (96h:28006)

[20] F. Morgan, Geometric measure theory A beginner's guide, Academic Press.

[21] Jani Onninen and Xiao Zhong, Mappings of finite distortion: a new proof for discreteness and openness, Proc. Roy. Soc. Edinburgh Sect. A 138 (2008), no. 5, 1097-1102, DOI 10.1017/S0308210506000825. MR2477453(2009k:30029)

[22] Kai Rajala, Remarks on the Iwaniec-Sverák conjecture, Indiana Univ. Math. J. 59 (2010), no. 6, 2027-2039, DOI 10.1512/iumj.2010.59.3946. MR2919747

[23] Seppo Rickman, Quasiregular mappings, Ergebnisse der Mathematik und ihrer Grenzgebiete (3) [Results in Mathematics and Related Areas (3)], vol. 26, Springer-Verlag, Berlin, 1993. MR 1238941 (95g:30026)

[24] S. Semmes, Finding curves on general spaces through quantitative topology, with applications to Sobolev and Poincaré inequalities, Selecta Math. (N.S.) 2 (1996), no. 2, 155-295, DOI 10.1007/BF01587936. MR1414889 (97j:46033)

[25] Nageswari Shanmugalingam, Newtonian spaces: an extension of Sobolev spaces to metric measure spaces, Rev. Mat. Iberoamericana 16 (2000), no. 2, 243-279, DOI 10.4171/RMI/275. MR:1809341 (2002b:46059)

[26] C. J. Titus and G. S. Young, The extension of interiority, with some applications, Trans. Amer. Math. Soc. 103 (1962), 329-340. MR0137103 (25 \#559)

[27] Jussi Väisälä, Minimal mappings in euclidean spaces, Ann. Acad. Sci. Fenn. Ser. A I No. 366 (1965), 22. MR0178454(31 \#2711)

Department of Mathematics and Statistics (P.O. Box 35 (MaD)), Fi-40014 University OF JYVÄSKYLÄ, FINLAND

E-mail address: ville.kirsila@jyu.fi 